\title{
Cosmological evolution in vector-tensor theories of gravity
}

\author{
José Beltrán Jiménez* and Antonio L. Maroto \\ Departamento de Física Teórica, Universidad Complutense de Madrid, 28040 Madrid, Spain
}

(Received 26 May 2009; published 8 September 2009)

\begin{abstract}
We present a detailed study of the cosmological evolution in general vector-tensor theories of gravity without potential terms. We consider the evolution of the vector field throughout the expansion history of the Universe and carry out a classification of models according to the behavior of the vector field in each cosmological epoch. We also analyze the case in which the Universe is dominated by the vector field, performing a complete analysis of the system phase map and identifying those attracting solutions which give rise to accelerated expansion. Moreover, we consider the evolution in a universe filled with a pressureless fluid in addition to the vector field and study the existence of attractors in which we can have a transition from matter domination to vector domination with accelerated expansion so that the vector field may play the role of dark energy. We find that the existence of solutions with late-time accelerated expansion is a generic prediction of vector-tensor theories and that such solutions typically lead to the presence of future singularities. Finally, limits from local gravity tests are used to get constraints on the value of the vector field at small (Solar System) scales.
\end{abstract}

DOI: 10.1103/PhysRevD.80.063512

PACS numbers: 98.80. $-\mathrm{k}, 95.36 .+\mathrm{x}$

\section{INTRODUCTION}

General relativity (GR) is considered nowadays the standard theory of the gravitational phenomena. In this theory, the gravitational interaction is mediated by a pure spin two field which allows one to describe the geometry of the background space-time where the physical processes take place. In spite of its elegance and experimental success, the possible existence of other metric theories of gravity compatible with Solar System experiments, as well as the importance of knowing the true theory of the gravitational interaction to describe properly relativistic astrophysical objects, motivated a long time ago the search for alternative gravitational theories with new degrees of freedom, in addition to the metric tensor. The first of these attempts was the so-called Brans-Dicke theory in which, apart from having the metric tensor, the gravitational interaction is described by a scalar field which makes the gravitational constant vary in space and time. Since the time when this new theory was proposed in the early 1960s, many other possibilities containing new degrees of freedom (not necessarily scalars) have been proposed.

In cosmology, the interest in alternatives to GR has grown recently because of the existence of several problems which cannot be satisfactorily addressed within the framework of GR without resorting to unknown components, namely, dark matter and dark energy. The need of new dark matter particles first appeared in order to account for the rotation curves of galaxies which seemed to indicate the existence of some kind of nonluminous matter in the haloes of such galaxies. However, its presence is also crucial to explain some cosmological observations as, for

\footnotetext{
*jobeltra@ fis.ucm.es

maroto@fis.ucm.es
}

instance, the formation of the structures that we observe today (galaxies, clusters, etc.).

The other dark component, dark energy, would be responsible for the accelerated expansion of the Universe first inferred from observations of distant type Ia supernovae [1] and subsequently confirmed by more precise supernovae measurements [2] as well as other cosmological probes, mainly the cosmic microwave background (CMB) temperature power spectrum and baryon acoustic oscillations [3]. Although it is possible to explain the accelerated expansion by introducing a cosmological constant term in Einstein's equations, this poses a problem from the theoretical point of view because its value, according to observations, is extremely small compared to the natural scale of gravity set by Newton's constant. Although this fact would not need to be a problem, the actual situation is that a theory with two scales differing in many orders of magnitude does not seem very natural, and that is why this problem is usually referred to as the naturalness problem. For this reason, many models trying to play the role of dynamical dark energy have been developed during the last decade and can be broadly classified in two classes: on one hand, models in which dark energy is a new field to add to the standard composition of the Universe [4] and, on the other hand, models in which the accelerated expansion would be an effect of a modified theory of gravity [5]. Nevertheless, this distinction is not always very clear because some modified gravity models require the introduction of new fields which, indeed, may play the role of dark energy.

One class of alternative theories of gravity with an extra field, in addition to the metric tensor, are the so-called vector-tensor theories, in which the gravitational action is modified by adding a vector field that is nonminimally coupled to gravity. The study of vector-tensor theories to 
describe the gravitational interaction as alternatives to GR started long ago with the works by Will, Nordtvedt, and Hellings [6] in the early 1970s as candidates to produce preferred frame effects. After these pioneering works, vector-tensor theories were abandoned because gravitational experiments seemed to rule out such preferred frame effects. Moreover, fluctuations of the vector field could be either timelike or spacelike so that those models were generally thought to present instabilities. A detailed treatment on this issue was developed in [7] for the case without a potential term and in [8] for the case with a potential term and it was shown that there is still some room in the parameter space for stable models. A special class of these theories reemerged due to the increasing interest in models with Lorentz violation [9]. The breaking of Lorentz invariance was achieved by the presence of a vector field whose norm was forced to be constant by means of a Lagrange multiplier. Moreover, some of these models are free of instabilities as was shown in [10].

More recently, after the discovery of the accelerated expansion of the Universe, vector-tensor theories supplemented with potential terms have got much attention as possible candidates for dark energy [11]. Nevertheless, vector-tensor theories even in the absence of potential terms have also turned out to be compelling candidates for dark energy [12] and they could even solve the aforementioned naturalness problem that most dark energy models exhibit. A remarkable example is the electromagnetic field. Indeed, it has been recently shown that electromagnetic quantum fluctuations generated during an inflationary epoch at the electroweak scale can give rise to an effective cosmological constant whose value is in agreement with the observed value [13]. Finally, vector fields have also been used for other cosmological purposes as candidates to drive an inflationary epoch [14], to generate nonsingular cosmologies [15], as dark matter candidates [16], and as candidates to solve some of the observed anomalies in the CMB power spectrum [17].

Given the increasing interest in vector-tensor theories of gravity in order to understand the wide variety of cosmological problems explained above, it seems useful to provide a detailed analysis of the cosmological evolution in a general vector-tensor theory so that one can know whether a particular theory will be able to play a determined role in the Universe's history. In this work, we shall focus on the problem of dark energy and obtain the necessary conditions to produce late-time acceleration, although the results obtained will be general enough to be useful in other cosmological contexts as well.

The paper is organized as follows: in Sec. II we describe the general vector-tensor theory and give the corresponding equations. Section III is devoted to study the evolution of the vector field in an isotropic Friedmann-RobertsonWalker universe. We shall give the behavior of the vector field in the different phases that the Universe has under- gone, namely: inflation, radiation domination, and matter domination. After that, in Sec. IV we shall study the case in which the temporal component of the vector field dominates the Universe and, in Sec. V, we shall obtain the region in the parameter space in which we might have attracting solutions with accelerated expansion. The case of a universe filled with matter plus the vector field will be studied in Sec. VI where it is shown that solutions exist which describe a transition from matter dominance to vector dominance with accelerated expansion. In Sec. VII we use current limits on local gravity tests to obtain constraints on the vector field at small scales.

\section{VECTOR-TENSOR THEORIES OF GRAVITY}

We shall start by writing the most general action for a vector-tensor theory without any other restriction apart from having second order linear equations of motion [18]:

$$
\begin{aligned}
S\left[g_{\mu \nu}, A_{\mu}\right]= & \int d^{4} x \sqrt{-g}\left[-\frac{1}{16 \pi G} R+\omega R A_{\mu} A^{\mu}\right. \\
& +\tilde{\sigma} R_{\mu \nu} A^{\mu} A^{\nu}+\tau \nabla_{\mu} A_{\nu} \nabla^{\mu} A^{\nu} \\
& \left.+\varepsilon F_{\mu \nu} F^{\mu \nu}\right]
\end{aligned}
$$

with $\omega, \tilde{\sigma}, \tau, \varepsilon$ dimensionless parameters, and $F_{\mu \nu}=$ $\partial_{\mu} A_{\nu}-\partial_{\nu} A_{\mu}$. In the so-called Aether-Einstein models, the vector field norm is fixed by introducing a Lagrange multiplier in the action of the form $\lambda_{m}\left(A_{\mu} A^{\mu} \pm m^{2}\right)$ so that $A_{\mu}$ is constrained to be either timelike or spacelike. In other cases, the vector field is supplied with a mass term or even more complicated potential terms. As we said above, throughout this work we shall focus on vector-tensor theories without Lagrange multipliers nor potential terms. Notice also that terms given in (1) are the only possibilities without introducing new scales in the theory and that give rise to linear equations of motion for the vector field. If such an action is just a low energy limit of some underlying theory, one would expect to have corrections involving terms of dimension higher than 4 which would be suppressed by some scale $M$.

For subsequent calculations we shall work with an alternative form of action (1) obtained via an integration by parts:

$$
\begin{aligned}
S\left[g_{\mu \nu}, A_{\mu}\right]= & \int d^{4} x \sqrt{-g}\left[-\frac{1}{16 \pi G} R+\omega R A_{\mu} A^{\mu}\right. \\
& \left.+\sigma R_{\mu \nu} A^{\mu} A^{\nu}+\lambda\left(\nabla_{\mu} A^{\mu}\right)^{2}+\epsilon F_{\mu \nu} F^{\mu \nu}\right],
\end{aligned}
$$

where the new parameters relate to the old ones as follows:

$$
\sigma=\tilde{\sigma}-\tau \quad \lambda=\tau \quad \epsilon=\frac{2 \varepsilon+\tau}{2} .
$$

We prefer the new form of the action because it allows a 
more suggestive interpretation for each term, namely: the $\epsilon$ term is nothing but the $U(1)$ gauge invariant kinetic term for the vector field, the $\lambda$ term is analogous to the gauge fixing term introduced in the electromagnetic quantization and, finally, both the $\omega$ and $\sigma$ terms are nonminimal couplings to gravity and play the role of effective mass terms for the vector field driven by gravity.

The gravitational equations obtained from action (2) by varying with respect to the metric tensor can be written in the following way:

$$
G_{\mu \nu}=8 \pi G\left(\omega T_{\mu \nu}^{\omega}+\sigma T_{\mu \nu}^{\sigma}+\lambda T_{\mu \nu}^{\lambda}+\epsilon T_{\mu \nu}^{\epsilon}+T_{\mu \nu}^{N G}\right),
$$

where $T_{\mu \nu}^{N G}$ is the energy-momentum tensor corresponding to other fields rather than $A_{\mu}$ (generally the inflaton, matter, and radiation) and we have defined

$$
\begin{aligned}
T_{\mu \nu}^{\omega}= & 2\left[\square A^{2} g_{\mu \nu}+A^{2} G_{\mu \nu}+R A_{\mu} A_{\nu}-\nabla_{\mu} \nabla_{\nu} A^{2}\right] \\
T_{\mu \nu}^{\sigma}= & g_{\mu \nu}\left[\nabla_{\alpha} \nabla_{\beta}\left(A^{\alpha} A^{\beta}\right)-R_{\alpha \beta} A^{\alpha} A^{\beta}\right]+\square\left(A_{\mu} A_{\nu}\right) \\
& -2 \nabla_{\alpha} \nabla_{(\mu}\left(A_{\nu)} A^{\alpha}\right)+4 A^{\alpha} A_{(\mu} R_{\nu) \alpha} \\
T_{\mu \nu}^{\lambda}= & g_{\mu \nu}\left[\left(\nabla_{\alpha} A^{\alpha}\right)^{2}+2 A^{\alpha} \nabla_{\alpha}\left(\nabla_{\beta} A^{\beta}\right)\right] \\
& -4 A_{(\mu} \nabla_{\nu)}\left(\nabla_{\alpha} A^{\alpha}\right) \\
T_{\mu \nu}^{\epsilon}= & 4 F_{\mu \alpha} F_{\nu}{ }^{\alpha}-g_{\mu \nu} F_{\alpha \beta} F^{\alpha \beta} \\
T_{\mu \nu}^{N G}= & \frac{2}{\sqrt{-g}} \frac{\delta S_{N G}}{\delta g^{\mu \nu}}
\end{aligned}
$$

with $\square=\nabla_{\mu} \nabla^{\mu}, A^{2}=A_{\mu} A^{\mu}$ and brackets in a pair of indices denoting symmetrization with respect to the corresponding indices.

Apart from the gravitational equations we can obtain a set of field equations for $A_{\mu}$ by varying the action with respect to the vector field to give

$$
2 \epsilon \nabla_{\nu} F^{\mu \nu}-\lambda \nabla^{\mu}\left(\nabla_{\nu} A^{\nu}\right)+\omega R A^{\mu}+\sigma R_{\nu}^{\mu} A^{\nu}=0 .
$$

As we are interested in the cosmological evolution of the vector field (especially as a candidate for dark energy) we shall focus on the simplest case in which the field is homogeneous, i.e., we shall study the evolution of the zero Fourier mode of the vector field. Actually, this will correspond to all Fourier modes whose physical wavelengths are much larger than the Hubble radius (superHubble modes). In fact, this is the relevant part of the field for the cosmological expansion evolution, although the inhomogeneous part could also be very important for the anisotropies of the CMB or for structure formation, but this is out of the scope of this work. Besides, we shall choose the spatial component of the field lying along the $z$ axis in such a way that we can write $A_{\mu}=\left(A_{0}(t), 0,0, A_{z}(t)\right)$ and, therefore, we have axial symmetry around that axis. Thus, the metric tensor will be appropriately described by that of the axisymmetric Bianchi type I space-time:

$$
d s^{2}=d t^{2}-a_{\perp}(t)^{2}\left(d x^{2}+d y^{2}\right)-a_{\|}(t)^{2} d z^{2},
$$

where $a_{\perp}$ and $a_{\|}$are the transverse and longitudinal scale factors, respectively. For this metric, the field equations read

$$
\begin{gathered}
\lambda\left[\ddot{A}_{0}+\left(2 H_{\perp}+H_{\|}\right) \dot{A}_{0}\right]+\left[(2 \omega+\sigma)\left(2 H_{\perp}^{2}+H_{\|}^{2}\right)\right. \\
\left.+(2 \omega+\sigma+\lambda)\left(2 \dot{H}_{\perp}+\dot{H}_{\|}\right)+2 \omega\left(2 H_{\perp} H_{\|}+H_{\perp}^{2}\right)\right] A_{0} \\
=0, \quad \text { (8) } \\
2 \epsilon\left[\ddot{A}_{z}+\left(2 H_{\perp}-H_{\|}\right) \dot{A}_{z}\right]+\left[2 \omega\left(3 H_{\perp}^{2}+2 \dot{H}_{\perp}\right)\right. \\
\left.+(2 \omega+\sigma)\left(\dot{H}_{\|}+H_{\|}^{2}+2 H_{\perp} H_{\|}\right)\right] A_{z}=0, \quad \text { (9) }
\end{gathered}
$$

where a dot stands for derivative with respect to the cosmic time $t$ and $H_{\|}=\dot{a}_{\|} / a_{\|}$and $H_{\perp}=\dot{a}_{\perp} / a_{\perp}$ are the longitudinal and transverse expansion rates, respectively. In these equations we see that the expansion of the Universe provides an effective mass for each component of the vector field as well as a friction term. It is interesting to note that the dynamics of $A_{0}$ and $A_{z}$ are driven by $\lambda$ and $\epsilon$, respectively, whereas the rest of parameters of the action, $\omega$ and $\sigma$, only affect the effective mass of the field. In fact, the presence of nonvanishing values for $\lambda$ and $\epsilon$ ensures the existence of evolving $A_{0}$ and $A_{z}$ respectively. On the contrary, if one of these parameters is zero, the corresponding component does not have dynamics and, in general, will vanish.

The highly isotropic CMB power spectrum that we observe today shows that the anisotropy at the last scattering surface was very small so that it is justified to consider small deviations from a pure isotropic universe so that

$$
a_{\perp}(t)=a(t) \quad a_{\|}(t)=a(t)\left(1+\frac{1}{2} h\right)
$$

with $h \ll 1$ and $a(t)$ the isotropic scale factor. Then, we can describe the anisotropy by means of the degree of anisotropy $h$ in terms of which the metric can be written as a perturbed Friedmann-Lemaitre-Robertson-Walker (FLRW) given by

$$
d s^{2}=d t^{2}-a(t)^{2}\left(\delta_{i j}+h_{i j}\right) d x^{i} d x^{j}
$$

with $h_{i j}=h \delta_{i z} \delta_{j z}$ and $a(t)$ the usual scale factor. Moreover, the degree of anisotropy can be related to the linearized Einstein tensor for the Bianchi type I metric as follows:

$$
G_{\perp}-G_{\|}=\frac{1}{2 a^{3}} \frac{d}{d t}\left(a^{3} \dot{h}\right)+\mathcal{O}\left(h^{2}\right),
$$

where $G_{\perp}=G_{x}^{x}=G_{y}^{y}$ and $G_{\|}=G_{z}^{z}$. Then, from Einstein equations we can obtain the evolution for the degree of anisotropy which happens to be

$$
h=16 \pi G \int \frac{1}{a^{3}}\left[\int a^{3}\left(p_{\|}-p_{\perp}\right) d t\right] d t .
$$

In principle, the problem has not been solved yet because the expression inside the integral will depend on $h$ as well. 
However, we can obtain an approximate solution by replacing such an integrand by its expression in the isotropic case. In fact, one could obtain more accurate solutions by an iterative process. Thus, if we now assume that the only source of anisotropy comes from the spatial component of the vector field we have that

$$
\begin{aligned}
a^{2}\left(p_{\|}-p_{\perp}\right)= & {\left[\left(24 \omega_{\epsilon}+12 \omega_{\epsilon} \sigma_{\epsilon}+8 \sigma_{\epsilon}+3 \sigma_{\epsilon}^{2}\right) H^{2}\right.} \\
& \left.+\left(12 \omega_{\epsilon}+6 \omega_{\epsilon} \sigma_{\epsilon}+4 \sigma_{\epsilon}+\sigma_{\epsilon}^{2}\right) \dot{H}\right] A_{z}^{2} \\
& +4 \sigma_{\epsilon} H A_{z} \dot{A}_{z}-2\left(2+\sigma_{\epsilon}\right) \dot{A}_{z}^{2},
\end{aligned}
$$

where $H=\dot{a} / a$ the Hubble expansion rate and we have introduced the notation $\omega_{\epsilon}=\omega / \epsilon$ and $\sigma_{\epsilon}=\sigma / \epsilon$. In the rest of this work we shall perform a detailed analysis of the isotropic evolution so that we could eventually use those results to evaluate (13) and, therefore, to discriminate those models in which the degree of anisotropy grows or decays as the Universe expands, that is, what models would give rise to large-scale anisotropies. For a detailed treatment on the anisotropy evolution in dark energy models see [19]. Moreover, the generated large-scale anisotropy would affect the photons coming from the last scattering surface so that it would give a new contribution to the low multipoles of the CMB. In fact, this can be used to rule out those models in which the new contribution is larger than the observed one.

To end this section we would like to comment on a very interesting feature of these models. In [20] it was shown that a dark energy field carrying a nonvanishing density of momentum could modify the usual interpretation of the CMB dipole as well as the value of the quadrupole. The density of momentum of dark energy can be interpreted as a relative motion of this component with respect to the others. Given that photons, baryons, and dark matter particles were strongly coupled in the early Universe, they originally shared a common large-scale rest frame so that, after becoming decoupled, they should remain at rest with respect to each other because of momentum conservation. However, the presence of a dark energy fluid with a nonvanishing density of momentum allows the existence of relative velocities among all the components without violating the momentum conservation. Therefore, as a vector field has spatial components one would expect it to carry density of momentum, essentially determined by $A_{i}$ and, as a consequence, it would be a natural candidate for a moving dark energy model. Nevertheless, once one uses the equations of motion it turns out that the density of momentum vanishes identically, i.e., $T^{0 i}=0$ over the field equations. Hence, although the vector field can provide largescale anisotropy supported by its spatial component, it does not modify the cosmic rest frame so that the rest of the fluids will generally share a common large-scale rest frame and no effects on the CMB dipole are expected. However, it is possible that vector perturbations could be supported by perturbations of the vector field so that, unlike in standard $\Lambda \mathrm{CDM}$, we could obtain large peculiar velocities at large scales as those observed in [21].

\section{EVOLUTION IN AN ISOTROPIC UNIVERSE}

In this section we shall write down and solve the equations for the vector field in a universe dominated by an isotropic perfect fluid, with the energy density of the vector field negligible. In such a case, both expansion factors become the same $a_{\perp}=a_{\|}=a$ as well as the expansion rates $H_{\perp}=H_{\|}=H$. This is equivalent to neglecting the effects of the small degree of anisotropy that may be present. In such a case, the field equations read

$$
\begin{array}{r}
\ddot{A}_{0}+3 H \dot{A}_{0}+\left[3\left(4 \omega_{\lambda}+\sigma_{\lambda}\right) H^{2}+3\left(1+2 \omega_{\lambda}+\sigma_{\lambda}\right) \dot{H}\right] A_{0} \\
=0 \\
\ddot{A}_{z}+H \dot{A}_{z}+\frac{1}{2}\left[3\left(4 \omega_{\epsilon}+\sigma_{\epsilon}\right) H^{2}+\left(6 \omega_{\epsilon}+\sigma_{\epsilon}\right) \dot{H}\right] A_{z}=0 .
\end{array}
$$

On the other hand, the energy density associated to $A_{0}$ and $A_{z}$ in the isotropic case are

$$
\begin{aligned}
\rho_{A_{0}}= & \lambda\left[3\left(3+2 \omega_{\lambda}+2 \sigma_{\lambda}\right) H^{2} A_{0}^{2}\right. \\
& \left.+6\left(1+2 \omega_{\lambda}+\sigma_{\lambda}\right) H A_{0} \dot{A}_{0}+\dot{A}_{0}^{2}\right] \\
\rho_{A_{z}}= & -\frac{2 \epsilon}{a^{2}}\left[-\left(3 \omega_{\epsilon}+\sigma_{\epsilon}\right) H^{2} A_{z}^{2}\right. \\
& \left.+\left(6 \omega_{\epsilon}+\sigma_{\epsilon}\right) H A_{0} \dot{A}_{0}+\dot{A}_{0}^{2}\right] .
\end{aligned}
$$

In these expressions we have introduced again the notation $\omega_{\lambda}=\omega / \lambda, \sigma_{\lambda}=\sigma / \lambda, \omega_{\epsilon}=\omega / \epsilon$, and $\sigma_{\epsilon}=\sigma / \epsilon$, which only makes sense for $\lambda \neq 0$ and $\epsilon \neq 0$. However, if that is not the case, the corresponding component does not have dynamics and, generally, vanishes so that it does not play any role, as we explained above.

Although we are restricting ourselves to the case of isotropic expansion, one should be aware of the fact that the presence of a small shear could modify the evolution of the spatial components of the vector field because, in the stability analysis around a FRW metric, there could be vanishing eigenvalues [22] and, as a consequence, the evolution of $A_{z}$ could be different from that determined by $(15)$.

In next subsections we shall study the evolution of the vector field in the different epochs of the expansion history of the Universe and carry out a classification of the models according to their behaviors.

\section{A. Inflationary (de Sitter) epoch}

During the inflationary era, the Universe undergoes an exponential expansion so that the Hubble parameter $H$ is constant, i.e., $a \propto e^{H t}$. In such a case, the solutions to (15) can be expressed as 


$$
\begin{aligned}
& A_{0}=\left(A_{0}^{+} e^{c_{0} H t}+A_{0}^{-} e^{-c_{0} H t}\right) e^{-3 H t / 2} \\
& A_{z}=\left(A_{z}^{+} e^{c_{z} H t}+A_{0}^{-} e^{-c_{z} H t}\right) e^{-H t / 2},
\end{aligned}
$$

where

$$
\begin{gathered}
c_{0}=\frac{1}{2} \sqrt{9-48 \omega_{\lambda}-12 \sigma_{\lambda}}, \\
c_{z}=\frac{1}{2} \sqrt{1-24 \omega_{\epsilon}-6 \sigma_{\epsilon}} .
\end{gathered}
$$

Then, the evolution of the temporal component depends on whether $c_{0}$ is real or complex. That way, we find that it oscillates with frequency $\left|c_{0}\right|$ and it is modulated by a damping factor of the form $e^{-3 H t / 2}$ for $16 \omega_{\lambda}+4 \sigma_{\lambda}>3$ whereas it evolves as $e^{\left(c_{0}-3 / 2\right) H t}$ for $16 \omega_{\lambda}+4 \sigma_{\lambda}<3$. When $16 \omega_{\lambda}+4 \sigma_{\lambda}=3$, we have that $c_{0}=0$ and the vector field evolves as $A_{0}=\left(C_{1}^{0}+C_{2}^{0} t\right) e^{-3 H t / 2}$.

Concerning $A_{z}$, it has an oscillating evolution with frequency $\left|c_{z}\right|$ suppressed by $e^{-H t / 2}$ for $24 \omega_{\lambda}+6 \sigma_{\lambda}>1$ and it evolves as $e^{\left(c_{z}-1 / 2\right) H t}$ for $24 \omega_{\lambda}+6 \sigma_{\lambda}<1$. Finally, for $24 \omega_{\lambda}+6 \sigma_{\lambda}=1$ we have that $c_{z}=0$ and the field evolves as $A_{z}=\left(C_{1}^{z}+C_{2}^{z} t\right) e^{-3 H t / 2}$.

When we insert solutions (17) into (16) we obtain the energy density evolutions, which can be written as

$$
\begin{aligned}
& \rho_{A_{0}}=\rho_{A_{0}^{+}} a^{2 c_{0}-3}+\rho_{A_{0}^{-}} a^{-2 c_{0}-3} \\
& \rho_{A_{z}}=\rho_{A_{z}^{+}} a^{2 c_{z}-3}+\rho_{A_{z}^{-}} a^{-2 c_{z}-3},
\end{aligned}
$$

where we have defined

$$
\begin{aligned}
\rho_{A_{0}^{ \pm}}= & \lambda\left[3\left(3+2 \omega_{\lambda}+2 \sigma_{\lambda}\right)+6\left(1+2 \omega_{\lambda}+\sigma_{\lambda}\right)\right. \\
& \left.\times\left( \pm c_{0}-3 / 2\right)+\left( \pm c_{0}-3 / 2\right)^{2}\right]\left(H A_{0}^{ \pm}\right)^{2} \\
\rho_{A_{z}^{ \pm}}= & -2 \epsilon\left[-\left(3 \omega_{\epsilon}+\sigma_{\epsilon}\right)+\left(6 \omega_{\epsilon}+\sigma_{\epsilon}\right)\left( \pm c_{z}-1 / 2\right)\right. \\
& \left.+\left( \pm c_{z}-1 / 2\right)^{2}\right]\left(H A_{z}^{ \pm}\right)^{2} .
\end{aligned}
$$

In these expressions we see that the temporal component is suppressed during inflation in models with $2 c_{0}<3 \Rightarrow$ $4 \omega_{\lambda}+\sigma_{\lambda}>0$ whereas an inflationary epoch amplifies $A_{0}$ for those models with $4 \omega_{\lambda}+\sigma_{\lambda}<0$. For $4 \omega_{\lambda}+\sigma_{\lambda}=$ 0 , the temporal component has constant energy density. Moreover, for the aforementioned special case with $c_{0}=0$ the energy density is given by

$$
\begin{aligned}
\left.\rho_{A_{0}}\right|_{c_{0}=0}= & \frac{\lambda}{2} C_{2}^{0}\left[3\left(5-8 \omega_{\lambda}\right) H C_{2}^{0} t-\left(24 \omega_{\lambda}-15\right) H C_{1}^{0}\right. \\
& \left.+2 C_{2}^{0}\right] a^{-3}
\end{aligned}
$$

so it decays as $\sim t a^{-3}$ (unless $5-8 \omega_{\lambda}=0$ ).

Analogously, we find that the spatial component is amplified during inflation in models with $12 \omega_{\epsilon}+3 \sigma_{\epsilon}<-4$, it is suppressed for those models with $12 \omega_{\epsilon}+3 \sigma_{\epsilon}>-4$, and it has constant energy density if the condition $12 \omega_{\epsilon}+$ $3 \sigma_{\epsilon}=-4$ is satisfied. Again, for the special case with $c_{z}=0$ we have a different evolution given by

$$
\begin{aligned}
\left.\rho_{A_{z}}\right|_{c_{z}=0}= & \frac{\epsilon}{3} C_{2}^{z}\left[\left(5-12 \omega_{\epsilon}\right) H C_{2}^{z} t-\left(12 \omega_{\epsilon}-5\right) H C_{1}^{z}\right. \\
& \left.+2 C_{2}^{z}\right] a^{-3},
\end{aligned}
$$

so it decays as $\sim t a^{-3}$ (unless $5-12 \omega_{\epsilon}=0$ ).

Finally, notice that the oscillating behavior of the field will translate into an oscillating evolution of the energy density so that in those cases in which the field oscillates the energy density for the corresponding component is suppressed by a factor $a^{-3}$. In fact, if the inflationary era lasts $N e$ folds, i.e., the scale factor increases as $a_{\text {end }} / a_{\text {in }}=$ $e^{N}$, we can calculate the amplification or suppression of the field at the end of inflation and that is given by

$$
\begin{aligned}
& \ln \left[\frac{A_{0}\left(t_{\text {end }}\right)}{A_{0}\left(t_{\text {in }}\right)}\right]=\left[\operatorname{Re}\left(c_{0}\right)-\frac{3}{2}\right] N \\
& \ln \left[\frac{A_{z}\left(t_{\text {end }}\right)}{A_{z}\left(t_{\text {in }}\right)}\right]=\left[\operatorname{Re}\left(c_{z}\right)-\frac{1}{2}\right] N .
\end{aligned}
$$

For the energy densities of each component we can proceed similarly to obtain

$$
\begin{aligned}
& \left.\ln \left[\frac{\rho_{A_{0}}\left(t_{\text {end }}\right)}{\rho_{A_{0}}\left(t_{\text {in }}\right)}\right]=\left[2 \operatorname{Re}\left(c_{0}\right)-3\right)\right] N \\
& \left.\ln \left[\frac{\rho_{A_{z}}\left(t_{\text {end }}\right)}{\rho_{A_{z}}\left(t_{\text {in }}\right)}\right]=\left[2 \operatorname{Re}\left(c_{z}\right)-3\right)\right] N .
\end{aligned}
$$

In these expressions, the real parts of $c_{0}$ and $c_{z}$ have to be taken because they are either real or purely imaginary and, in the latter case, the only effect of $c_{0, z}$ is to produce oscillations, but neither suppression nor amplification of the vector field, as we commented above. In the special cases with $c_{0, z}=0$ we have to add a term $\ln \frac{t_{\text {end }}}{t_{\text {in }}}$ on the righthand sides of the above expressions, although such a term is usually much smaller than $N$ and can be safely neglected.

\section{B. Barotropic fluid domination}

In a universe dominated by a barotropic perfect fluid with constant equation of state $w=p / \rho$, the scale factor evolves according to a power law of the form $a \propto t^{p}$ with $p=\frac{2}{3(1+w)}$ so that $H=p / t$. In such a case, the field equations (15) have the following solutions:

$$
A_{0}(t)=A_{0}^{+} t^{\alpha_{+}}+A_{0}^{-} t^{\alpha_{-}} \quad A_{z}(t)=A_{z}^{+} t^{\beta_{+}}+A_{z}^{-} t^{\beta_{-}}
$$

with 


$$
\begin{gathered}
\alpha_{ \pm}=\frac{1}{2}\left[1-3 p \pm \sqrt{1+6\left(1+4 \omega_{\lambda}+2 \sigma_{\lambda}\right) p+3\left(3-16 \omega_{\lambda}-4 \sigma_{\lambda}\right) p^{2}}\right] \\
\beta_{ \pm}=\frac{1}{2}\left[1-p \pm \sqrt{1+2\left(6 \omega_{\epsilon}+\sigma_{\epsilon}-1\right) p-\left(24 \omega_{\epsilon}+6 \sigma_{\epsilon}-1\right) p^{2}}\right] .
\end{gathered}
$$

As in the inflationary epoch, we see that the components of the vector field have two different types of evolution depending on whether $\alpha_{ \pm}$and $\beta_{ \pm}$are real or complex. Thus, if the term inside the root is positive, the corresponding component of the field will evolve as a power law essentially given by the growing mode whereas if the term inside the root is negative, the field will oscillate with an amplitude proportional to $t^{(1-3 p) / 2}$ for $A_{0}$ or $t^{(1-p) / 2}$ for $A_{z}$. In fact, for $\alpha, \beta \in \mathbb{C}$, the solutions of the vector field can be expressed as:

$$
\begin{aligned}
& A_{0}(t)=t^{\operatorname{Re}(\alpha)}\left[C_{1}^{0} \cos (\operatorname{Im}(\alpha) \ln t)+C_{2}^{0} \cos (\operatorname{Im}(\alpha) \ln t)\right] \\
& A_{z}(t)=t^{\operatorname{Re}(\beta)}\left[C_{1}^{z} \cos (\operatorname{Im}(\beta) \ln t)+C_{2}^{z} \cos (\operatorname{Im}(\beta) \ln t)\right],
\end{aligned}
$$

where we see that the vector field actually oscillates harmonically in $\ln t$ and not in the proper time $t$. There is still another special case which is that when we have degeneration in the solutions, i.e., when $\alpha_{+}=\alpha_{-}$or $\beta_{+}=\beta_{-}$. If any of these relations takes place, the corresponding component of the vector field has a logarithmic solution in addition to the potential solution, being the complete solution as follows:

$$
\begin{aligned}
& A_{0}=\left(C_{1}^{0}+C_{2}^{0} \ln t\right) t^{(1-3 p) / 2} \\
& A_{z}=\left(C_{1}^{z}+C_{2}^{z} \ln t\right) t^{(1-p) / 2} .
\end{aligned}
$$

The evolutions of the energy densities are achieved by inserting solutions (30) into (16) and, for $\alpha, \beta \in \mathbb{R}$, are given by

$$
\begin{aligned}
& \rho_{A_{0}}=\rho_{A_{0}^{+}} a^{\kappa_{0}^{+}}+\rho_{A_{0}^{-}} a^{\kappa_{0}^{-}} \\
& \rho_{A_{z}}=\rho_{A_{z}^{+}} a^{\kappa_{z}^{+}}+\rho_{A_{z}^{-}} a^{\kappa_{z}^{-}},
\end{aligned}
$$

where

$$
\begin{aligned}
\rho_{A_{0}^{ \pm}}= & \lambda\left[3\left(3+2 \omega_{\lambda}+2 \sigma_{\lambda}\right) p^{2}\right. \\
+ & \left.6\left(1+2 \omega_{\lambda}+\sigma_{\lambda}\right) p \alpha_{ \pm}+\alpha_{ \pm}^{2}\right]\left(A_{0}^{ \pm}\right)^{2} \\
\rho_{A_{z}^{ \pm}}= & -2 \epsilon\left[-\left(3 \omega_{\epsilon}+\sigma_{\epsilon}\right) p^{2}\right. \\
& \left.+\left(6 \omega_{\epsilon}+\sigma_{\epsilon}\right) p \beta_{ \pm}+\beta_{ \pm}^{2}\right]\left(A_{z}^{ \pm}\right)^{2}
\end{aligned}
$$

and

$$
\kappa_{0}^{ \pm}=2 \frac{\alpha_{ \pm}-1}{p} \quad \kappa_{z}^{ \pm}=2 \frac{\beta_{ \pm}-1-p}{p} .
$$

Again, given that $\kappa_{0, z}^{+} \geq \kappa_{0, z}^{-}$, the energy densities will evolve proportionally to $a^{\kappa_{0, z}^{+}}$so that the vector field be- haves as the superposition of two perfect fluids with equations of state $w_{0, z}=-\frac{1}{3}\left(1+\kappa_{0, z}^{+}\right)$. However, in some cases, it might happen that $\rho_{A_{0, z}^{+}}$vanishes and, as a consequence, the corresponding energy density will evolve as $a^{\kappa_{0, z}^{-}}$rather than $a^{\kappa_{0, z}^{+}}$.

When $\alpha_{ \pm}$and $\beta_{ \pm}$are complex, the energy densities still evolve as $\rho_{A_{0, z}}=\rho_{A_{0, z}^{+}} a^{\kappa_{0, z}^{+}}$although we must replace $\alpha_{+} \rightarrow \operatorname{Re}\left(\alpha_{+}\right)$and $\beta_{+} \rightarrow \operatorname{Re}\left(\beta_{+}\right)$in (39) and $\rho_{A_{0, z}^{+}}$are oscillating functions instead of constants.

For the degenerate case with a logarithmic solution, the energy densities are given by

$$
\begin{aligned}
\left.\rho_{A_{0}}\right|_{\alpha_{+}=\alpha_{-}}= & \lambda \frac{\left(C_{2}^{0}\right)^{2}}{2(1-p)}\left[\left[1-2 p+\left(24 \omega_{\lambda}-15\right) p^{2}\right]\right. \\
& \left.\times\left(\frac{C_{1}^{0}}{C_{2}^{0}}+\ln t\right)+2(1-p)\right] a^{\kappa_{0}} \\
\left.\rho_{A_{z}}\right|_{\beta_{+}=\beta_{-}}= & \epsilon \frac{\left(C_{2}^{z}\right)^{2}}{(1-3 p)}\left[\left[-1+6 p+\left(12 \omega_{\epsilon}-5\right) p^{2}\right]\right. \\
& \left.\times\left(\frac{C_{1}^{z}}{C_{2}^{z}}+\ln t\right)+2(3 p-1)\right] a^{\kappa_{z}} .
\end{aligned}
$$

Therefore, the evolution is the same as in the nondegenerate case modified by a logarithmic variation.

In any case, we can carry out a classification of the models according to whether the energy density of each component grows, decays, or remains constant. Finally, we can also identify scaling behaviors or whether the energy density of the vector field grows or decays with respect to that of the dominant component. In Figs. 1 and 2 we show the evolution in the different regions in the parameter space.

\section{Radiation dominated epoch}

In the radiation dominated epoch we have that $p=1 / 2$ and the evolution of the vector field, according to (32), is given by

$$
\begin{gathered}
\alpha_{ \pm}^{R}=-\frac{1}{4}\left[1 \mp \sqrt{25+12 \sigma_{\lambda}}\right] \\
\beta_{ \pm}^{R}=\frac{1}{4}\left[1 \pm \sqrt{1-2 \sigma_{\epsilon}}\right] .
\end{gathered}
$$

These expressions allow one to find that the temporal component evolves as a power law for $\sigma_{\lambda}>-\frac{25}{12}$ whereas it oscillates with an amplitude decaying as $t^{-1 / 4}$ for $\sigma_{\lambda}<$ $-\frac{25}{12}$. The degenerate case happens for $\sigma_{\lambda}=-\frac{25}{12}$. For the 


\begin{tabular}{|c|c|c|c|}
\hline Property & Inflation & Radiation & Matter \\
\hline Oscillating & $4 \omega_{\lambda}+\sigma_{\lambda}>\frac{3}{4}$ & $\sigma_{\lambda}<-\frac{25}{12}$ & $2 \omega_{\lambda}-\sigma_{\lambda}>\frac{27}{8}$ \\
\hline Decaying & $0<4 \omega_{\lambda}+\sigma_{\lambda}<\frac{3}{4}$ & $-\frac{25}{12}<\sigma_{\lambda}<-2$ & $3<2 \omega_{\lambda}-\sigma_{\lambda}<\frac{27}{8}$ \\
\hline Scaling & $4 \omega_{\lambda}+\sigma_{\lambda}=0$ & $\sigma_{\lambda}=-2$ & $2 \omega_{\lambda}-\sigma_{\lambda}=3$ \\
\hline Growing & $4 \omega_{\lambda}+\sigma_{\lambda}<0$ & $\sigma_{\lambda}>-2$ & $2 \omega_{\lambda}-\sigma_{\lambda}<3$ \\
\hline Constant & $4 \omega_{\lambda}+\sigma_{\lambda}=0$ & $\sigma_{\lambda}=0$ & $2 \omega_{\lambda}-\sigma_{\lambda}=0$ \\
\hline
\end{tabular}
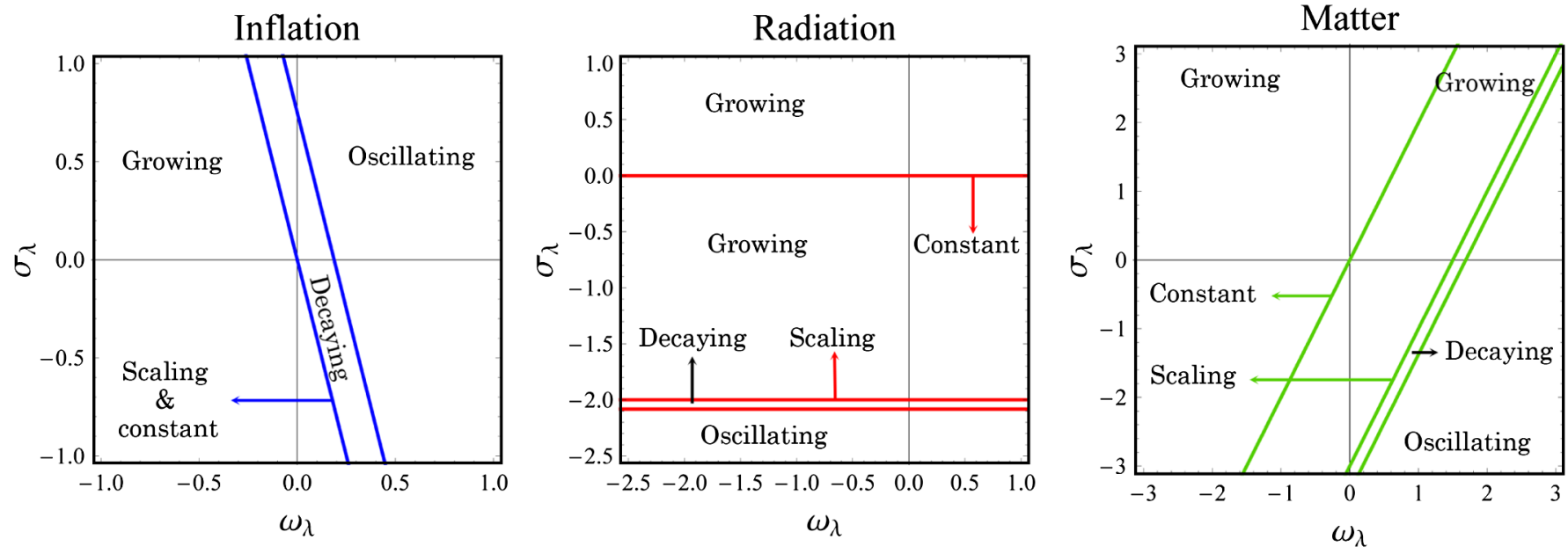

FIG. 1 (color online). Behavior of the temporal component of the vector field in the different phases of the Universe's history according to the values of the parameters of the model. The labels Decaying and Growing refer to the behavior with respect to the dominant component, whereas Scaling means that it evolves in the same way as the background. 


\begin{tabular}{|c||c|c|c|}
\hline Property & Inflation & Radiation & Matter \\
\hline \hline Oscillating & $4 \omega_{\epsilon}+\sigma_{\epsilon}>\frac{1}{6}$ & $\sigma_{\epsilon}>\frac{1}{2}$ & $2 \omega_{\epsilon}+\sigma_{\epsilon}>\frac{1}{12}$ \\
\hline \multirow{2}{*}{ Decaying } & $-\frac{4}{3}<4 \omega_{\epsilon}+\sigma_{\epsilon}<\frac{1}{6}$ & $0<\sigma_{\epsilon}<1$ & $-\frac{2}{3}<2 \omega_{\epsilon}+\sigma_{\epsilon}<\frac{1}{12}$ \\
\hline Scaling & $4 \omega_{\epsilon}+\sigma_{\epsilon}=-\frac{4}{3}$ & $\sigma_{\epsilon}=0$ & $2 \omega_{\epsilon}+\sigma_{\epsilon}=-\frac{2}{3}$ \\
\hline Growing & $4 \omega_{\epsilon}+\sigma_{\epsilon}<-\frac{4}{3}$ & $\sigma_{\epsilon}<0$ & $2 \omega_{\epsilon}+\sigma_{\epsilon}<-\frac{2}{3}$ \\
& & & \\
\hline & & & \\
\hline & & & \\
\hline
\end{tabular}
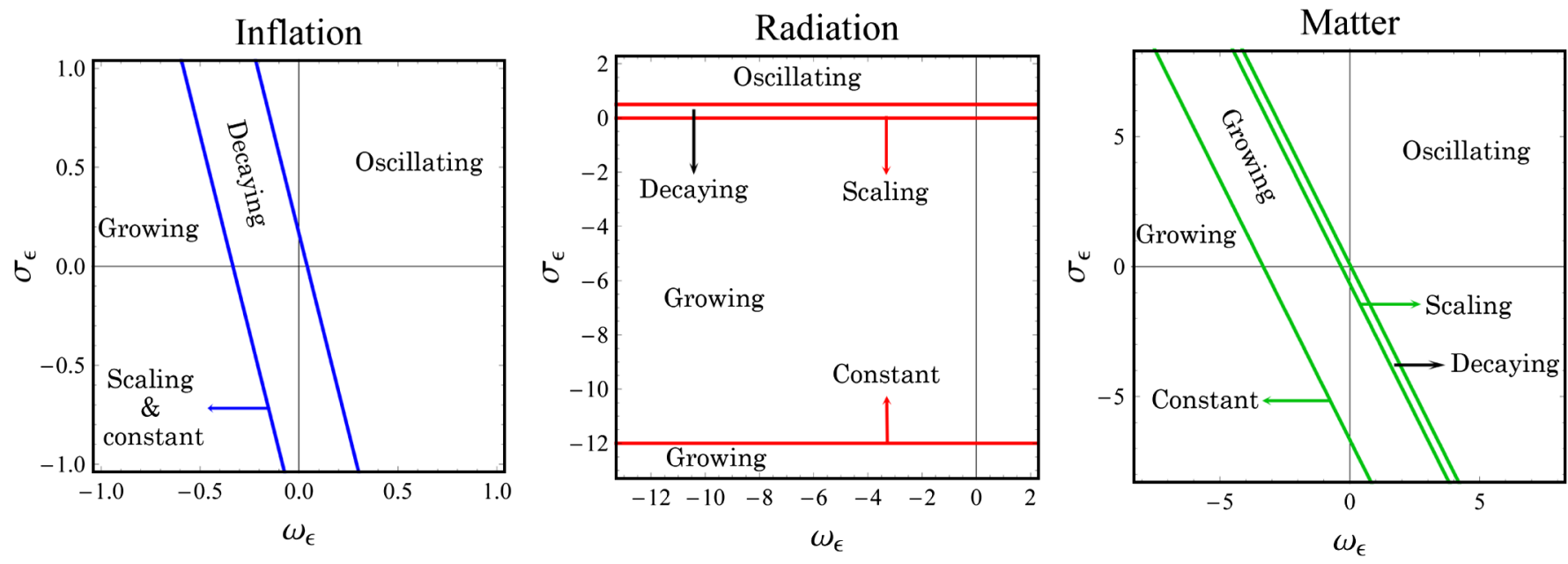

FIG. 2 (color online). Behavior of the spatial component of the vector field in the different phases of the Universe's history according to the values of the parameters of the model. The labels Decaying and Growing refer to the behavior with respect to the dominant component, whereas Scaling means that it evolves in the same way as the background. 
spatial component of the vector field, we have power law behavior for $\sigma_{\epsilon}<\frac{1}{2}$ and it oscillates with an amplitude growing as $t^{1 / 4}$ for $\sigma_{\epsilon}>\frac{1}{2}$, whereas the logarithmic solution appears for $\sigma_{\epsilon}=\frac{1}{2}$. Notice that the evolution of the vector field does not depend on the parameter $\omega$ of the action, which is due to the fact that the Ricci scalar $R$ vanishes in a universe dominated by radiation.

Concerning the evolution of the energy densities, according to (39), we have that

$$
\begin{gathered}
\kappa_{0 \pm}^{R}=-5 \pm \sqrt{25+12 \sigma_{\lambda}} \\
\kappa_{z \pm}^{R}=-5 \pm \sqrt{1-2 \sigma_{\epsilon}} .
\end{gathered}
$$

If we want the vector field to be dominated by its temporal component we must impose the condition $\kappa_{0+}>$ $\kappa_{z+}$, which leads to the constraint $6 \sigma_{\lambda}+\sigma_{\epsilon}>-12$. That way we prevent the generation of large-scale anisotropy that would be in conflict with observations.

The temporal component of the vector field will have constant energy density in this epoch for $\kappa_{0+}^{R}=0$ which is satisfied by the model with $\sigma_{\lambda}=0$ and it has scaling evolution if $\kappa_{0+}^{R}=-4$ which happens for $\sigma_{\lambda}=-2$. From this last condition we also find that, in models with $\sigma_{\lambda}>-2$, the energy density associated to the temporal component grows with respect to that of the radiation fluid $\rho_{R}$ whereas in those models with $\sigma_{\lambda}<-2$, the ratio $\rho_{A_{0}} / \rho_{R}$ decays as the Universe expands. See Fig. 1 for a summary of these behaviors.

Concerning the spatial component, the condition of constant energy density is satisfied for models with $\sigma_{\epsilon}=$ -12 whereas it scales as radiation in models with $\sigma_{\epsilon}=0$. Finally, the energy density associated to the spatial component of the vector field grows (decays) with respect to $\rho_{R}$ in models with $\sigma_{\epsilon}<0\left(\sigma_{\epsilon}>0\right)$. This classification is shown in Fig. 2.

\section{Matter dominated epoch}

In a universe dominated by a pressureless fluid the scale factor evolves as $a \propto t^{2 / 3}$ so that

$$
\begin{gathered}
\alpha_{ \pm}^{M}=-\frac{1}{6}\left[3 \mp \sqrt{81-48 \omega_{\lambda}+24 \sigma_{\lambda}}\right] \\
\beta_{ \pm}^{M}=\frac{1}{6}\left[1 \pm \sqrt{1-24 \omega_{\epsilon}-12 \sigma_{\epsilon}}\right] .
\end{gathered}
$$

In this case, the temporal component oscillates with amplitude proportional to $t^{-1 / 2}$ for $16 \omega_{\lambda}-8 \sigma_{\lambda}>27$, it evolves with a power law for $16 \omega_{\lambda}-8 \sigma_{\lambda}<27$, and the degenerate case corresponds to $16 \omega_{\lambda}-8 \sigma_{\lambda}=27$. On the other hand, $A_{z}$ follows a power law evolution for $24 \omega_{\epsilon}+$ $12 \sigma_{\epsilon}<1$, it oscillates with amplitude proportional to $t^{1 / 6}$ for $24 \omega_{\epsilon}+12 \sigma_{\epsilon}>1$, and the degenerate case happens for $24 \omega_{\epsilon}+12 \sigma_{\epsilon}=1$.

Moreover, $\kappa_{0 \pm}^{R}$ and $\kappa_{z \pm}^{R}$ become

$$
\begin{gathered}
\kappa_{0 \pm}^{M}=\frac{1}{2}\left[-9 \pm \sqrt{81-48 \omega_{\lambda}+24 \sigma_{\lambda}}\right] \\
\kappa_{z \pm}^{M}=\frac{1}{2}\left[-9 \pm \sqrt{1-24 \omega_{\epsilon}-12 \sigma_{\epsilon}}\right] .
\end{gathered}
$$

In this epoch, the condition for the temporal contribution to dominate over the spatial one reads $6\left(\omega_{\epsilon}-2 \omega_{\lambda}\right)+$ $3\left(\sigma_{\epsilon}-2 \sigma_{\lambda}\right)>-80$.

The scaling behavior for the energy density associated to the temporal component in this epoch is obtained from the condition $\kappa_{0+}^{M}=-3$, whose solution is $2 \omega_{\lambda}-\sigma_{\lambda}=3$. Moreover, for $2 \omega_{\lambda}-\sigma_{\lambda}<3$ the energy density of the vector field grows with respect to that of matter and, as a consequence, the Universe is eventually dominated by it. Notice that this is a necessary condition to have a dark energy model, i.e., those models with $2 \omega_{\lambda}-\sigma_{\lambda}>3$ will never dominate the energy content of the Universe if there is a matter component and, as a consequence, it cannot be responsible for the present acceleration. Figure 1 shows this classification.

For the spatial component we obtain constant energy density in models with $2 \omega_{\epsilon}+\sigma_{\epsilon}=-20 / 3$ and scaling evolution for $2 \omega_{\epsilon}+\sigma_{\epsilon}=-2 / 3$. Finally, the energy density of $A_{z}$ grows (decays) with respect to $\rho_{M}$ in models with $2 \omega_{\epsilon}+\sigma_{\epsilon}<-2 / 3\left(2 \omega_{\epsilon}+\sigma_{\epsilon}>-2 / 3\right)$. This is shown in Fig. 2.

\section{VECTOR DOMINANCE}

In this section we shall study the case in which the Universe becomes dominated by the temporal component of the vector field so that the anisotropy is small and we can use the isotropic equations. Thus, we have the field equation for $A_{0}$ given in (15) and the two Einstein equations

$$
\begin{gathered}
3 H^{2}=8 \pi G \rho_{A_{0}} \\
3 H^{2}+2 \dot{H}=-8 \pi G p_{A_{0}} .
\end{gathered}
$$

Although, of course, only two of the three equations are independent, it will be useful to work with all of them.

For the subsequent analysis, it will be convenient to introduce the field variable $x \equiv \frac{d \ln A_{0}}{d \ln a}$ so that we can obtain the following autonomous system:

$$
\frac{d H}{d N}=3 \frac{\left(2 \omega_{\lambda}+\sigma_{\lambda}\right) x^{2}-2\left(4 \omega_{\lambda}+\sigma_{\lambda}\right) x-3\left(4 \omega_{\lambda}+\sigma_{\lambda}\right)\left(2 \omega_{\lambda}+\sigma_{\lambda}+1\right)}{\left[x+3\left(2 \omega_{\lambda}+\sigma_{\lambda}+1\right)\right]^{2}} H
$$




$$
\frac{d x}{d N}=-\frac{x^{2}+6\left(2 \omega_{\lambda}+\sigma_{\lambda}+1\right) x+3\left(2 \omega_{\lambda}+2 \sigma_{\lambda}+3\right)}{x+3\left(2 \omega_{\lambda}+\sigma_{\lambda}+1\right)} x
$$

with $N=\ln a$. These two equations can be combined to give the following equation for the trajectories in the phase map:

$$
\frac{d H}{d x}=-3 \frac{\left(2 \omega_{\lambda}+\sigma_{\lambda}\right) x^{2}-2\left(4 \omega_{\lambda}+\sigma_{\lambda}\right) x-3\left(4 \omega_{\lambda}+\sigma_{\lambda}\right)\left(2 \omega_{\lambda}+\sigma_{\lambda}+1\right)}{\left[x^{2}+6\left(2 \omega_{\lambda}+\sigma_{\lambda}+1\right) x+3\left(2 \omega_{\lambda}+2 \sigma_{\lambda}+3\right)\right]\left[x+3\left(2 \omega_{\lambda}+\sigma_{\lambda}+1\right)\right]} \frac{H}{x} .
$$

This equation can be readily integrated for given values of the parameters, although we shall not do it, but we shall study the phase map and, from its features, we shall obtain the relevant information.

In addition to the equations given above, we have the following constraint provided by the Friedmann equation

$$
\frac{1}{3} \lambda A_{0}^{2}\left[x^{2}+6\left(2 \omega_{\lambda}+\sigma_{\lambda}+1\right) x+3\left(2 \omega_{\lambda}+2 \sigma_{\lambda}+3\right)\right]=1 .
$$

This relation constrains the possible values of $x$ because the condition

$$
\lambda\left[x^{2}+6\left(1+\sigma_{\lambda}+2 \omega_{\lambda}\right) x+3\left(3+2 \sigma_{\lambda}+2 \omega_{\lambda}\right)\right] \geq 0
$$

must hold. This condition restricts the physically admissible trajectories as those for which the vector field carries positive energy density and can always be achieved by means of a suitable choice of the sign of $\lambda$. Notice that all the dependency of the problem on the parameter $\lambda$ is indeed contained in this condition.

In (53) we see that the equation $\frac{d H}{d N}=0$ has two solutions, $H=0$ and $x=x_{l}^{ \pm}$, with

$$
x_{l}^{ \pm}=\frac{4 \omega_{\lambda}+\sigma_{\lambda} \pm \sqrt{\left(4 \omega_{\lambda}+\sigma_{\lambda}\right)\left[2\left(5 \omega_{\lambda}+2 \sigma_{\lambda}\right)+3\left(2 \omega_{\lambda}+\sigma_{\lambda}\right)^{2}\right]}}{2 \omega_{\lambda}+\sigma_{\lambda}},
$$

whereas the solutions of $\frac{d x}{d N}=0$ are $x=0$ and $x=x_{c}^{ \pm}$, with

$$
x_{c}^{ \pm}=-3\left(2 \omega_{\lambda}+\sigma_{\lambda}+1\right) \pm \sqrt{6\left(5 \omega_{\lambda}+2 \sigma_{\lambda}\right)+9\left(2 \omega_{\lambda}+\sigma_{\lambda}\right)^{2}} .
$$

Therefore, the autonomous system has generally three critical points: $P_{0}=(0,0)$ and $P_{ \pm}=\left(x_{c}^{ \pm}, 0\right)$. Moreover, when the equality $x_{c}^{ \pm}=x_{l}^{ \pm}$takes place, we have a critical line instead of a critical point because both $\frac{d H}{d N}$ and $\frac{d x}{d N}$ vanish regardless the value of $H$. Notice that the critical points $P_{ \pm}$only exist when the constraint $6\left(5 \omega_{\lambda}+2 \sigma_{\lambda}\right)+$ $9\left(2 \omega_{\lambda}+\sigma_{\lambda}\right)^{2} \geq 0$ is satisfied, which corresponds to the white region shown in Fig. 3. Moreover, the trajectories in the phase map have vertical tangents in the lines $x=0$ and $x=x_{c}^{ \pm}$so that, apart from being critical points, they are vertical separatrices (see Fig. 4). However, these are not the only separatrices in the phase map, but we have another vertical one in $x_{s}=-3\left(2 \omega_{\lambda}+\sigma_{\lambda}+1\right)$ as well as one horizontal one in the horizontal axis $H=0$. One interesting feature of the phase map is that $x_{s}=\left(x_{c}^{+}+x_{c}^{-}\right) / 2$, i.e., the separatrix at $x_{s}$ is always located in the middle of the two critical points. Notice that the critical points $x_{c}^{ \pm}$also separate the region with physically admissible trajectories according to (56) which imposes the energy density of the vector field to be positive, although to identify each of these regions we need to specify the sign of $\lambda$. According to the previous discussion, the phase map will be divided in several rectangular regions parallel to the axis which, in- deed, are disconnected from each other, i.e., the trajectories will not be able to cross from one to another. However, the particular picture will depend on the particular values of the parameters, making it possible to distinguish the following cases (see Fig. 4).

(1) Case I: The critical points $x_{c}^{ \pm}$exist and are different from each other, which imposes the condition $9\left(2 \omega_{\lambda}+\sigma_{\lambda}+1\right)^{2}-3\left(2 \omega_{\lambda}+2 \sigma_{\lambda}+3\right)>0$ and corresponds to the gray region in Fig. 3. Moreover, in this case we still have three different possibilities:

(a) Case Ia: Both critical points are different from zero and they do not coincide with $x_{s}$. In this case we have 4 vertical separatrices and the phase map is divided into 10 disconnected regions.

(b) Case Ib: One of the two critical points is zero. This case happens when $2 \omega_{\lambda}+2 \sigma_{\lambda}+3=0$ but $2 \omega_{\lambda}+$ $\sigma_{\lambda}+1 \neq 0$ so that they are not zero simultaneously. In fact, if $2 \omega_{\lambda}+\sigma_{\lambda}+1$ is positive (negative), then $x_{c}^{+}\left(x_{c}^{-}\right)$is at the origin. Notice that this simply says that, given that the separatrix located at $x_{s}$ is in the middle of $x_{-}$and $x_{+}$, the critical point that is at the origin depends on the sign of $x_{s}$. In this case we only have 3 vertical asymptotes and, as a 


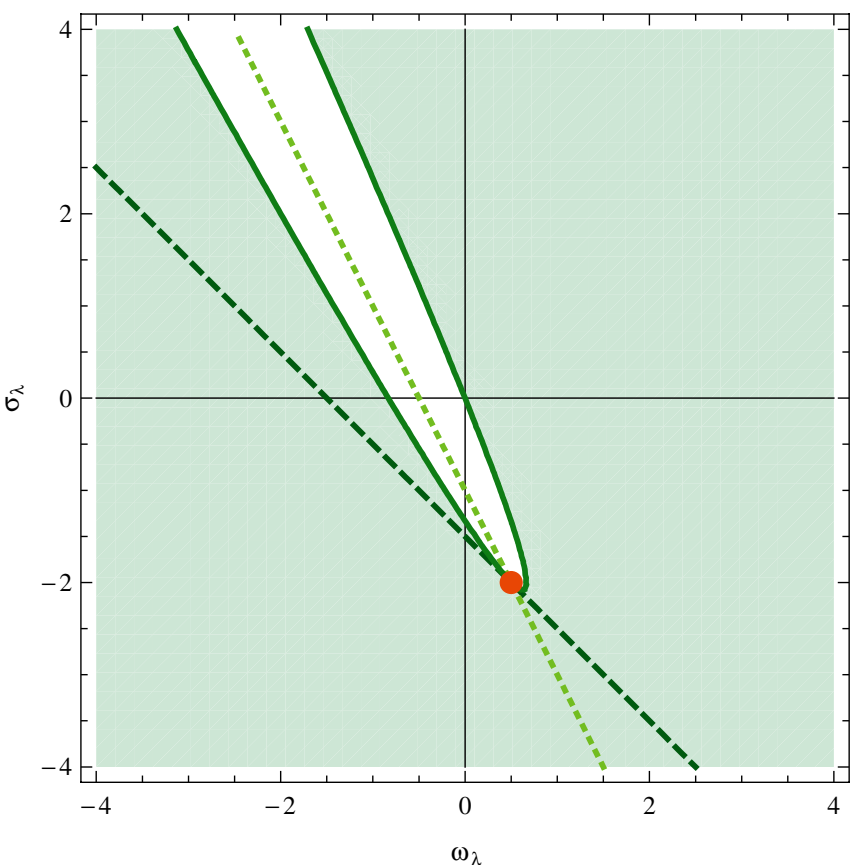

consequence, the phase map contains 8 regions. This case corresponds to the dashed line in Fig. 3, which represents the equation $2 \omega_{\lambda}+2 \sigma_{\lambda}+3=0$.

(c) Case Ic: The vertical separatrix satisfies $x_{s}=0$ so that the two critical points are symmetric with respect to the origin. The condition $x_{s}=0$ reads in
FIG. 3 (color online). This plot shows the different cases explained in the main text in the parameter space. The shaded (green) region corresponds to models in which both $x_{ \pm}$exist (case I) whereas in the white region neither of them is present (case II). The solid line identifies the models for which $x_{+}=x_{-}$ corresponding to case III. The dashed line (whose equation is $2 \omega_{\lambda}+2 \sigma_{\lambda}+3=0$ ) represents those models that have either $x_{-}$ or $x_{+}$at the origin (case $\mathrm{Ib}$ ) and the models whose parameters lie on the dotted line (with equation $2 \omega_{\lambda}+\sigma_{\lambda}+1=0$ ) have the separatrix $x_{s}$ at the origin. Thus, in the region above (below) that line $x_{s}$ is negative (positive). Case Ic corresponds to the piece of the dotted line inside the white region whereas the piece of the dotted line inside the gray region corresponds to case IIb. Finally, the (orange) dot with parameters $\omega_{\lambda}=1 / 2, \sigma_{\lambda}=-2$ gives the case IIIb in which $x_{ \pm}=x_{s}=0$.

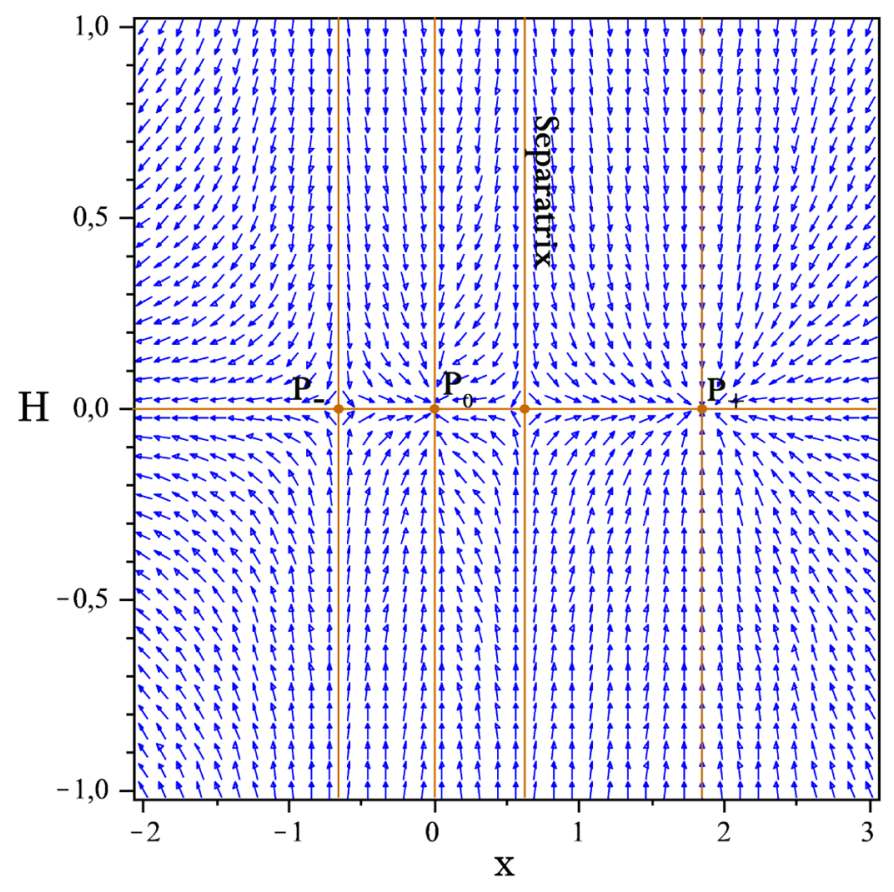

terms of the parameters $2 \omega_{\lambda}+\sigma_{\lambda}+1=0$ and, as a consequence, the critical point $P_{0}$ is absent. In this case we also have 3 vertical asymptotes and 8 regions in the phase map and this case corresponds to the dotted line inside the grey region in Fig. 3.

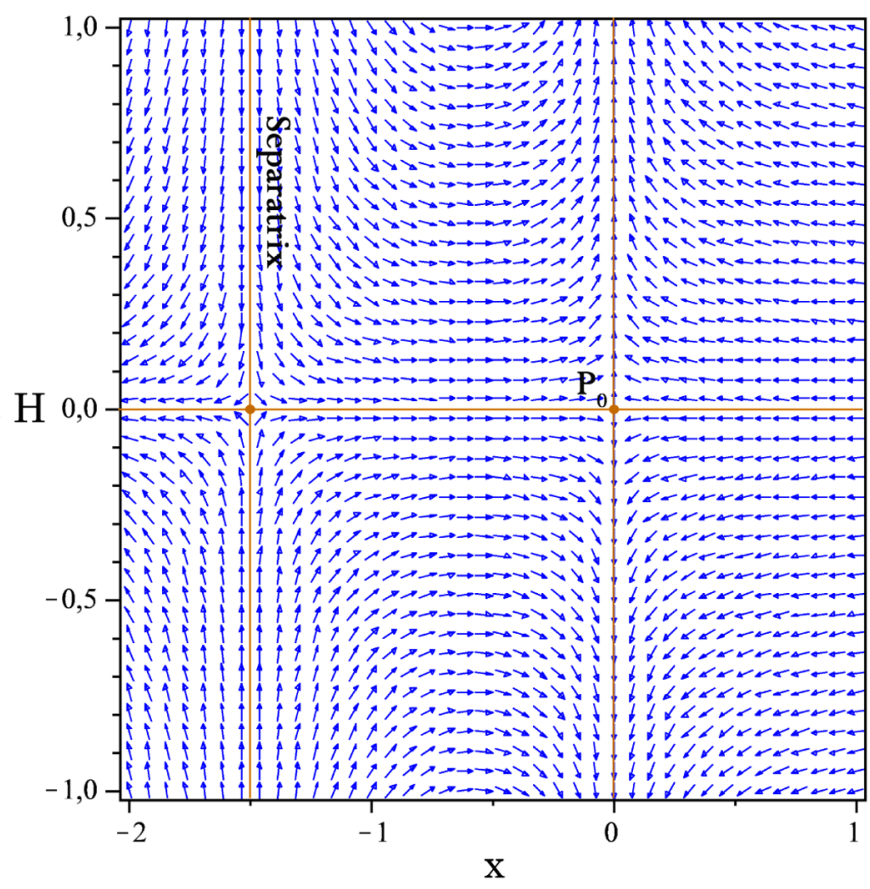

FIG. 4 (color online). These plots show two examples of phase maps for the autonomous system describing a universe dominated by the vector field. The left panel corresponds to a model lying in the gray region in Fig. 3 in which the two critical points $P_{ \pm}$are present whereas the right panel shows the phase map for a model in which these two critical points do not exist. We can see that all the critical points as well as the separatrix are vertical tangents and the $x$ axis is a horizontal separatrix, as explained in the main text. 
(2) Case II: Neither of the two critical points $x_{c}^{ \pm}$exists. This case satisfies $9\left(2 \omega_{\lambda}+\sigma_{\lambda}+1\right)^{2}-3\left(2 \omega_{\lambda}+\right.$ $\left.2 \sigma_{\lambda}+3\right)>0$ and corresponds to the white region in Fig. 3. Again, we have several subcases:

(a) Case IIa: The separatrix $x_{s}$ is different from zero. Then, we have 2 different vertical asymptotes and the phase map contains 6 regions.

(b) Case IIb: The separatrix is located at the origin $x_{s}=$ 0 so that $2 \omega_{\lambda}+\sigma_{\lambda}+1=0\left(P_{0}\right.$ is not a critical point) and we are left with just 1 vertical asymptote that divides the phase map in 4 regions. This case corresponds to the dotted line inside the white region in Fig. 3.

(3) Case III: The 2 critical points become equal so we only have 1 critical point which, indeed, coincides with $x_{s}$, i.e., $x_{c}^{+}=x_{c}^{-}=x_{s}=-3\left(2 \omega_{\lambda}+\sigma_{\lambda}+1\right)$. This case is represented by the solid line in Fig. 3. The 2 possibilities we have in this case are:

(a) Case IIIa: The critical point is different from zero so that we have 2 vertical asymptotes and the phase map contains 6 regions.

(b) Case IIIb: The critical point is zero. Then we only have 1 vertical separatrix and 4 regions. This case corresponds to the particular model $\left(\sigma_{\lambda}=-2\right.$, $\omega_{\lambda}=1 / 2$ ) and is represented by the (orange) dot in Fig. 3.

Now that we know the arrangement of the phase map for the different models attending to the existence and location of the critical points, we shall study the particular features of each critical point according to the values of the parameters:

(1) $P_{0}=(0,0)$. The eigenvalues for this critical point are

$$
\begin{aligned}
\mu_{H} & =-\frac{4 \omega_{\lambda}+\sigma_{\lambda}}{2 \omega_{\lambda}+\sigma_{\lambda}+1}, \\
\mu_{x} & =-\frac{2 \omega_{\lambda}+2 \sigma_{\lambda}+3}{2 \omega_{\lambda}+\sigma_{\lambda}+1} .
\end{aligned}
$$

Thus, we have that the critical point is a saddle point for models whose parameters are between the lines $4 \omega_{\lambda}+\sigma_{\lambda}=0$ and $2 \omega_{\lambda}+2 \sigma_{\lambda}+3=0$ whereas it is an attractor node if the parameters are in the external region. Notice that this critical point does not exist when $2 \omega_{\lambda}+\sigma_{\lambda}+1=0$ - that corresponds to case Ic in which the separatrix $x_{s}$ is placed at the origin. When $4 \omega_{\lambda}+\sigma_{\lambda}=0$ we have that $\mu_{H}=0$ and the critical point becomes a critical line because $x=0$ is a singular point irrespective of the value of $H$.

(2) $P_{ \pm}=\left(x_{c}^{ \pm}, 0\right)$. For these critical points the eigenvalues can be expressed as $\mu_{H}=-\left(2 x_{c}^{ \pm}+3\right)$ and $\mu_{x}=-2 x_{c}^{ \pm}$. Then, if the critical point is positive we have an attractor node whereas it behaves as a repelling node if $x_{c}^{ \pm}<-3 / 2$. Finally, in the range $-3 / 2<x_{c}^{ \pm}<0$, we get a saddle point. These ranges correspond to the regions in the parameter space shown in Fig. 5. The eigenvalues $\mu_{x}^{ \pm}$vanish when the corresponding critical point is located at the origin, i.e., $2 \sigma_{\lambda}+2 \omega_{\lambda}+3=0$. Then, we can have three different cases depending on the sign of $2 \omega_{\lambda}+\sigma_{\lambda}+1$, namely:

(a) $2 \omega_{\lambda}+\sigma_{\lambda}+1>0$. In this case $\mu_{x}^{+}=0$ and $\mu_{x}^{-}=$ $-6\left(2 \omega_{\lambda}+\sigma_{\lambda}+1\right)<0$.

(b) $2 \omega_{\lambda}+\sigma_{\lambda}+1=0$. This is the case IIIb described above in which both critical points are located at the origin and, as a consequence, $\mu_{x}^{+}=\mu_{x}^{-}=0$.

(c) $2 \omega_{\lambda}+\sigma_{\lambda}+1>0$. This case is the opposite to (a) above, i.e., $\mu_{x}^{-}=0$ and $\mu_{x}^{+}=-6\left(2 \omega_{\lambda}+\sigma_{\lambda}+\right.$ 1) $>0$.

On the other hand, the eigenvalues $\mu_{H}^{ \pm}$vanish in the case that the critical points are such that $x_{c}^{ \pm}=-3 / 2$ which is satisfied for models with $\sigma_{\lambda}+4 \omega_{\lambda}=3 / 4$. In this case we also obtain three possibilities in terms of the sign of $12 \omega_{\lambda}-15 / 2$, as follows:

(a) $12 \omega_{\lambda}-15 / 2>0$. In this case we have $\mu_{H}^{-}=0$ and $\mu_{H}^{+}=24 \omega_{\lambda}-15>0$.

(b) $12 \omega_{\lambda}-15 / 2=0$. In this case we have $\mu_{H}^{-}=$ $\mu_{H}^{+}=0$.

(c) $12 \omega_{\lambda}-15 / 2<0$. In this case we have $\mu_{H}^{+}=0$ and $\mu_{H}^{-}=24 \omega_{\lambda}-15<0$.

(3) Separatrix $x=x_{s}$. For values of $x$ close to $x_{s}$, i.e., $x=x_{s}+\delta$ with $\delta \rightarrow 0$ the equations become

$$
\begin{aligned}
& \frac{d H}{d N} \simeq \mu_{s} \frac{H}{\delta^{2}} \\
& \frac{d x}{d N} \simeq-\mu_{s} \frac{1}{\delta}
\end{aligned}
$$

with

$$
\begin{aligned}
\mu_{s}= & 9\left(2 \omega_{\lambda}+\sigma_{\lambda}+1\right)\left[12 \omega_{\lambda}^{2}+\sigma_{\lambda}\left(4+3 \sigma_{\lambda}\right)\right. \\
& \left.+2 \omega_{\lambda}\left(5+6 \sigma_{\lambda}\right)\right] .
\end{aligned}
$$

Then, the separatrix will attract the trajectories of the phase map for models in which $\mu_{s}>0$ whereas for models with $\mu_{s}<0$ the trajectories will go away from $x_{s}$. Note that this is true for both sides of the separatrix.

(4) $x \rightarrow \pm \infty$. In this case we have that $\frac{d x}{d N} \sim-x^{2}$ so that the trajectories will always approach from $+\infty$ and will move away to $-\infty$, i.e., the region with large values of $x+\infty$ repels the trajectories of the phase map whereas the trajectories become attracted by the region with $x \rightarrow-\infty$ irrespective of the values of the parameters in the action. This means that the region with large positive values of $x$ is always unstable for any choice of $\omega_{\lambda}$ and $\sigma_{\lambda}$ and, on the contrary, the region with negative large values of $x$ is always stable. 

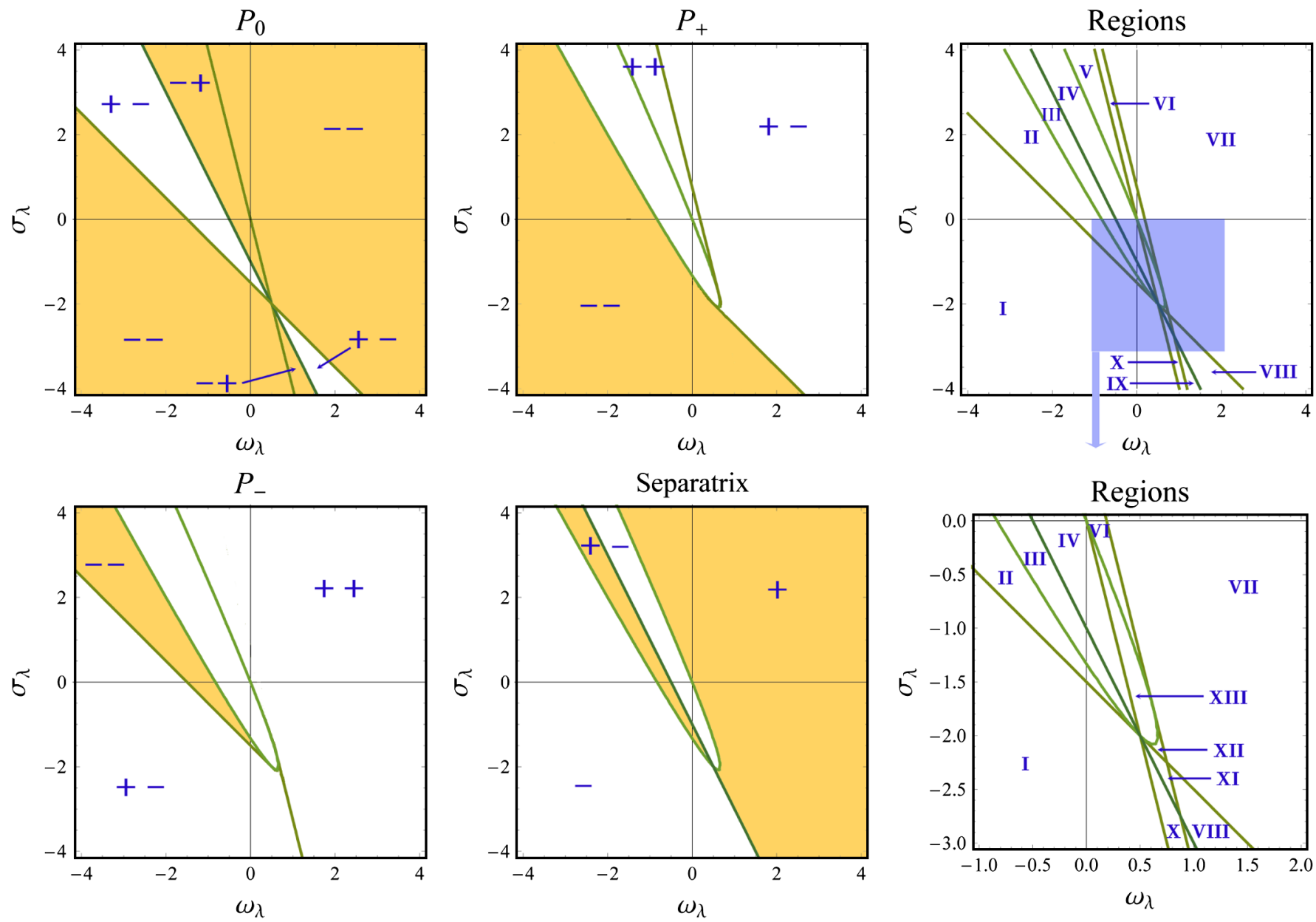

FIG. 5 (color online). In this plot we show the different regions obtained in the parameter space according to the features of the critical points. We have shaded (orange) the regions where the eigenvalue of $x$ is negative so that the trajectories in the phase map approach the corresponding point. We have also indicated the sign of each eigenvalue in the form $\left(\mu_{x}, \mu_{H}\right)$. For the separatrix we indicate the regions where the trajectories approach the separatrix. Finally, the two plots on the right show the 13 regions explained in the text (and summarized in Table I). The bottom-right plot corresponds to a zoom of the shaded (blue) region in the top-right panel above.

\section{A. Accelerating solutions}

In this section we shall enumerate the necessary conditions for a vector-tensor model to lead to accelerating solutions. To that end we shall express the equation of state in terms of the field variable $x$ :

$$
w=-\frac{\left(4 \omega_{\lambda}+2 \sigma_{\lambda}+1\right) x^{2}-2\left(2 \omega_{\lambda}-\sigma_{\lambda}-3\right) x-3\left(2 \omega_{\lambda}+\sigma_{\lambda}+1\right)\left(2 \omega_{\lambda}-\sigma_{\lambda}-3\right)}{\left[x+3\left(2 \omega_{\lambda}+\sigma_{\lambda}+1\right)\right]^{2}} .
$$

Notice that this equation of state only depends on $x$ and not on the Hubble parameter $H$. The models with accelerating solutions will be those in which $w$ evolves toward $w<$ $-1 / 3$. Moreover, as this work is intended to find models in which the vector field could play the role of dark energy, we shall demand that the accelerated phase is an attractor. To that end, we shall look at the equation of state for all the possible attracting places in the phase map as well as in the repelling ones. Notice that, as the equation of state does not depend on $H$ but only on $x$ we only need to require attractor or repelling properties with respect to $x$. For instance, if a critical point is a saddle point but with the trajectories going toward $x \rightarrow x_{\text {crit }}\left(\mu_{x}<0\right)$, it will be considered as an attractor and the opposite for a repelling point. Now, we shall study the existence of accelerating regimes in the phase map.

(1) $P_{0}$. As we pointed out above, this critical point will be an attractor in models with $\mu_{x}<0$. On the other 
hand, the equation of state for this critical point is

$$
w_{P_{0}}=\frac{2 \omega_{\lambda}-\sigma_{\lambda}-3}{3\left(2 \omega_{\lambda}+\sigma_{\lambda}+1\right)} .
$$

From this expression we see that $P_{0}$ corresponds to an accelerated phase $\left(w_{P_{0}}<-1 / 3\right)$ if the following condition holds:

$$
\frac{2 \omega_{\lambda}-1}{2 \omega_{\lambda}+\sigma_{\lambda}+1}<0
$$

which is satisfied for models in which either $1<$ $2 \omega_{\lambda}<-\left(1+\sigma_{\lambda}\right)$ or $-\left(1+\sigma_{\lambda}\right)<2 \omega_{\lambda}<1$. The corresponding region is shown in Fig. 6 and, in that figure, we see that there exists a region in which $P_{0}$ is an attractor and gives rise to accelerated expansion simultaneously.

(2) $P_{+}$. For these critical points, the equation of state becomes

$$
\begin{aligned}
w_{P_{ \pm}}= & -\left(8 \omega_{\lambda}+4 \sigma_{\lambda}+3\right) \pm \frac{4}{3} \\
& \times \sqrt{6\left(5 \omega_{\lambda}+2 \sigma_{\lambda}\right)+9\left(2 \omega_{\lambda}+\sigma_{\lambda}\right)^{2}} .
\end{aligned}
$$

Notice that if the condition of accelerated expansion is satisfied for $P_{+}$, then it is also satisfied for $P_{-}$ since $w_{P_{+}} \geq w_{P_{-}}$. The models that lead to accelerated expansion are shown in Fig. 6. However, in the same figure, we see that neither of the critical points $P_{ \pm}$behaves as an attractor in the region where we get accelerated expansion.

(3) Separatrix $x=x_{s}$. In this case the equation of state will evolve either to $+\infty$ or to $-\infty$ as the trajectory approaches the separatrix. The interesting case here is when $w \rightarrow-\infty$ so that we get acceleration. For $x=x_{s}+\delta$ with $\delta \rightarrow 0$, the equation of state becomes

$$
w_{s} \simeq-\frac{2 \mu_{s}}{3 \delta^{2}} .
$$
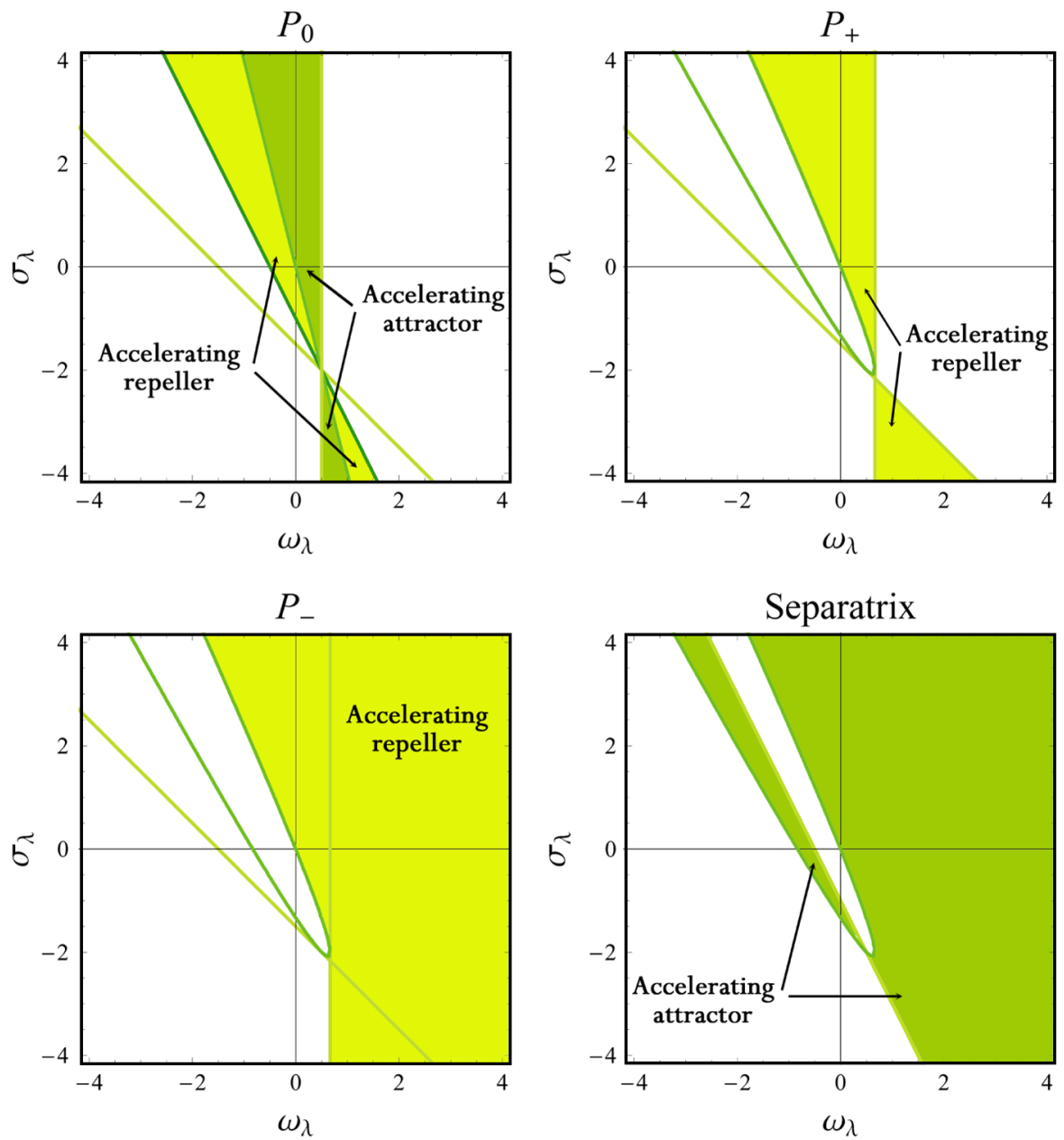

FIG. 6 (color online). In this plot we show the regions in which we get accelerated solutions in a universe dominated by the vector field for each critical point. The darkest shaded regions are those in which the accelerated solutions are attractors. We can see that neither for $P_{+}$nor for $P_{-}$can we obtain attracting accelerated solutions. 
In Fig. 6 we show the region in which the equation of state goes to $-\infty$, corresponding to the condition $\mu_{s}>0$. Notice that this condition also guarantees that the separatrix behaves as an attractor so all the cases in which the sepratrix is an attractor give rise to accelerated expansion.

(4) $x \rightarrow \pm \infty$. As we showed above, $x=-\infty$ is always an attractor whereas $x=+\infty$ is always a repelling point. Moreover, as $x$ approaches $\pm \infty$ the equation of state is given by

$$
w(x \rightarrow \pm \infty)=-\left(4 \omega_{\lambda}+2 \sigma_{\lambda}+1\right)
$$

so that it gives rise to accelerated expansion in models with $2 \omega_{\lambda}+\sigma_{\lambda}>-1 / 3$. This case is interesting for the vector field to drive an inflationary era because it could start with a large value of $x$ and, as $x=\infty$ repels the trajectories, it would be forced to evolve toward smaller values of $x$ until it reaches either $P_{+}$or $P_{0}$. Moreover, such an evolution can lead to accelerated expansion as long as the condition $2 \omega_{\lambda}+\sigma_{\lambda}>-1 / 3$ holds.

After having obtained the general conditions necessary to have accelerated expansion, we shall study the particular solutions in which the scale factor evolves as a power of the cosmic time, i.e., $a \propto t^{p}$, and we shall get some analytical solutions. For this expansion law, the vector field evolves according to (30), although, in this case, the parameter $p$ must be determined from the Einstein equations. When we introduce (30) in (50) we find that the vector field must take a constant value given by

$$
A_{0}^{\infty}=\frac{ \pm 1}{\sqrt{8 \pi G \lambda\left(3+2 \omega_{\lambda}+2 \sigma_{\lambda}\right)}} .
$$

Notice that this value only makes sense for $\lambda\left(3+2 \omega_{\lambda}+\right.$ $\left.2 \sigma_{\lambda}\right)>0$, which can always be fulfilled by a suitable choice of the parameter $\lambda$. Moreover, we also find that $p$ is given by

$$
p=\frac{1+2 \omega_{\lambda}+\sigma_{\lambda}}{4 \omega_{\lambda}+\sigma_{\lambda}} .
$$

With this expression we can calculate the deceleration parameter

$$
q_{\infty} \equiv-\frac{\ddot{a} a}{\dot{a}^{2}}=\frac{2 \omega_{\lambda}-1}{2 \omega_{\lambda}+\sigma_{\lambda}+1} .
$$

This deceleration parameter must be negative in order to have an accelerated expansion, but this is indeed the same condition that we found above in (65) when studying the critical point $P_{0}$. This was expected because the Hubble expansion rate goes to zero for a power law expansion and this imposes that $A_{0}$ has to be constant which means that $x=0$. Thus, we have just obtained nothing but the analytical solutions for the trajectories approaching $P_{0}$ along the $H$ axis. Notice that this is, indeed, the only critical point attracting trajectories with accelerated expansion and having a finite value for the vector field equation of state.

\section{TRANSITION FROM MATTER DOMINATION TO VECTOR DOMINATION: LATE-TIME ACCELERATED SOLUTIONS}

In previous sections we have studied a universe completely dominated by the vector field and obtained the necessary conditions to have accelerated solutions. In this section, however, we shall consider the case in which the Universe contains matter in addition to the vector field and study the circumstances under which we can get a transition from a matter dominated universe to an accelerated phase provided by the vector field so that the vector field can play the role of dark energy. To do so, we shall proceed as in previous sections, i.e., we shall obtain the corresponding autonomous system and identify attracting solutions in which the vector field eventually dominates the energy content of the Universe and has equation of state smaller than $-1 / 3$.

Before going on with the study of the autonomous system, we recall that a necessary condition to have a candidate to dark energy is that the energy density associated to the vector field decays slower than that of a pressureless fluid in the matter dominated epoch. This requirement guarantees the dominance of the vector field at late times so that it can drive the expansion of the Universe. According to Table I, such models are those whose parameters satisfy the condition

$$
2 \omega_{\lambda}-\sigma_{\lambda}<3 .
$$

We shall take this condition as a necessary requirement for the model to be able to play the role of dark energy.

The system of equations must be modified by introducing the matter contribution to the Friedmann equation so that

$$
3 H^{2}=8 \pi G\left(\rho_{A_{0}}+\rho_{M}\right) .
$$

Moreover, we have a new equation provided by the energy conservation of the matter fluid

$$
\dot{\rho}_{M}+3 H \rho_{M}=0 .
$$

As we did in the previous sections, we shall introduce the field variable $x \equiv \frac{d \ln A_{0}}{d \ln a}$ and the matter energy density will be described by the density parameter $\Omega_{M} \equiv \frac{\rho_{M}}{3 H^{2}}$. In terms of these variables, we can obtain the following autonomous system:

$$
\frac{d H}{d N}=3 \frac{\left(2 \omega_{\lambda}+\sigma_{\lambda}\right) x^{2}-2\left(4 \omega_{\lambda}+\sigma_{\lambda}\right) x-3\left(4 \omega_{\lambda}+\sigma_{\lambda}\right)\left(2 \omega_{\lambda}+\sigma_{\lambda}+1\right)+F_{H} \Omega_{M}}{\left[x+3\left(2 \omega_{\lambda}+\sigma_{\lambda}+1\right)\right]^{2}-\left[9\left(2 \omega_{\lambda}+\sigma_{\lambda}\right)^{2}+6\left(5 \omega_{\lambda}+2 \sigma_{\lambda}\right)\right] \Omega_{M}} H
$$


TABLE I. In this table we summarize the features of the phase map for the different regions shown in Fig. 5. When a given critical point is a saddle point we give the sign of the eigenvalue corresponding to $x$ so that we can know whether the trajectories approach the critical point (negative eigenvalue) or move away from it (positive eigenvalue) along the $x$ direction.

\begin{tabular}{lcccc}
\hline \hline Region & \multicolumn{1}{c}{$P_{0}$} & $P_{-}$ & Separatrix & $P_{+}$ \\
\hline I & Attractor node & Saddle point $\mu_{x}>0$ & Repelling & Attractor node \\
II & Saddle point $\mu_{x}>0$ & Attractor node & Repelling & Attractor node \\
III & Saddle point $\mu_{x}>0$ & & Attractor & Repelling \\
IV & Saddle point $\mu_{x}<0$ & & Attractor & Repelling node \\
V & Saddle point $\mu_{x}<0$ & Repelling node & Attractor & Repelling node \\
VI & Attractor node & Repelling node & Attractor & Saddle point $\mu_{x}>0$ \\
VII & Attractor node & Repelling node & Attractor node \\
VIII & Saddle point $\mu_{x}>0$ & Repelling node & Attracting & Attractor node \\
IX & Saddle point $\mu_{x}<0$ & Repelling node & Repelling & Attractor node \\
X & Saddle point $\mu_{x}<0$ & Repelling & Attractor node \\
XI & Saddle point $\mu_{x}>0$ & Saddle point $\mu_{x}>0$ & Attractor & Saddle point $\mu_{x}>0$ \\
XII & Attractor node & Saddle point $\mu_{x}>0$ & Attractor & Repelling \\
XIII & Attractor node & & & \\
\hline \hline
\end{tabular}

$$
\begin{aligned}
\frac{d x}{d N} & =-\frac{\left[x^{2}+6\left(2 \omega_{\lambda}+\sigma_{\lambda}+1\right) x+3\left(2 \omega_{\lambda}+2 \sigma_{\lambda}+3\right)\right]\left[x\left(x+3\left(2 \omega_{\lambda}+\sigma_{\lambda}+1\right)\right)+F_{x} \Omega_{M}\right]}{\left[x+3\left(2 \omega_{\lambda}+\sigma_{\lambda}+1\right)\right]^{2}-\left[9\left(2 \omega_{\lambda}+\sigma_{\lambda}\right)^{2}+6\left(5 \omega_{\lambda}+2 \sigma_{\lambda}\right)\right] \Omega_{M}} \\
\frac{d \Omega_{M}}{d N} & =3 \frac{\left[\left(4 \omega_{\lambda}+2 \sigma_{\lambda}+1\right) x^{2}-\left(4 \omega_{\lambda}-2 \sigma_{\lambda}-6\right) x-3\left(2 \omega_{\lambda}+\sigma_{\lambda}+1\right)\left(2 \omega_{\lambda}-\sigma_{\lambda}-3\right)\right]}{\left[x+3\left(2 \omega_{\lambda}+\sigma_{\lambda}+1\right)\right]^{2}-\left[9\left(2 \omega_{\lambda}+\sigma_{\lambda}\right)^{2}+6\left(5 \omega_{\lambda}+2 \sigma_{\lambda}\right)\right] \Omega_{M}}\left(1-\Omega_{M}\right) \Omega_{M},
\end{aligned}
$$

where we have defined

$$
\begin{aligned}
F_{H} & =-\frac{1}{2}\left[\left(4 \omega_{\lambda}+2 \sigma_{\lambda}+1\right) x^{2}-2\left(2 \omega_{\lambda}-\sigma_{\lambda}-3\right) x+6\left(4 \omega_{\lambda}+\sigma_{\lambda}\right)\left(2 \omega_{\lambda}+\sigma_{\lambda}\right)+9\left(2 \omega_{\lambda}-1\right)\right] \\
F_{x} & =-\frac{3}{2}\left[\left(4 \omega_{\lambda}+2 \sigma_{\lambda}+1\right) x-2 \omega_{\lambda}+\sigma_{\lambda}+3\right] .
\end{aligned}
$$

These equations are supplemented by the following constraint provided by the Friedmann equation:

$$
\frac{1}{3} \lambda A_{0}^{2}\left[x^{2}+6\left(2 \omega_{\lambda}+\sigma_{\lambda}+1\right) x+3\left(2 \omega_{\lambda}+2 \sigma_{\lambda}+3\right)\right]+\Omega_{M}=1 .
$$

As before, this relation will determine the sign of the parameter $\lambda$ in order to fulfill the condition $\lambda\left[x^{2}+6\left(2 \omega_{\lambda}+\sigma_{\lambda}+\right.\right.$ 1) $\left.x+3\left(2 \omega_{\lambda}+2 \sigma_{\lambda}+3\right)\right]>0$.

The equation of state for the vector field is given in this case by

$$
w=-\frac{\left(4 \omega_{\lambda}+2 \sigma_{\lambda}+1\right) x^{2}-2\left(2 \omega_{\lambda}-\sigma_{\lambda}-3\right) x-3\left(2 \omega_{\lambda}+\sigma_{\lambda}+1\right)\left(2 \omega_{\lambda}-\sigma_{\lambda}-3\right)}{\left[x+3\left(2 \omega_{\lambda}+\sigma_{\lambda}+1\right)\right]^{2}-\left[9\left(2 \omega_{\lambda}+\sigma_{\lambda}\right)^{2}+6\left(5 \omega_{\lambda}+2 \sigma_{\lambda}\right)\right] \Omega_{M}} .
$$

The sections $\left\{x, \Omega_{M}\right\}$ of the phase map do not depend on $H$. Notice that, as expected, the equations for $\frac{d H}{d N}$ and $\frac{d x}{d N}$ reduce to (53) when $\Omega_{M}=0$ so that all the critical points analyzed in the previous sections are also critical points of (76) with $\Omega_{M}=0$, which is the interesting case here because this means that the vector fields eventually dominate the energy of the Universe. Apart from these critical points with $\Omega_{M}=0$ we also have critical points with $\Omega_{M}=1$ which correspond to situations where matter drives the Universe expansion. As we are interested in obtaining solutions leading to late-time accelerated expansion driven by the vector field, we shall study just the critical points with $\Omega_{M}=0$ so that the critical values of $x$ and $H$ are the same as those studied in the vector dominance case. However, the features of the critical points may change because of the presence of matter. Therefore, for each critical point, we shall identify the necessary conditions for the corresponding critical point to be an attractor with respect to $\Omega_{M}$ and $x$ and, then, compute the equation of state in the critical point. This is possible because the equations for $\Omega_{M}$ and $x$ do not depend on $H$. Notice that the condition for $\Omega_{M}=0$ to be an attractor will, in general, differ from the condition given in (72) obtained by imposing that the energy density of the vector field grows with respect to that of matter. This is so because to achieve that condition we assumed that the 
amount of matter was initially dominant with respect to that of the vector field and, however, for $\Omega_{M}=0$ to be an attractor, such a condition does not need to be satisfied. Finally, it is interesting to remark that the region with $\Omega_{M}>0$ is disconnected from that with $\Omega_{M}<0$ because the trajectories are always tangent to the plane $\Omega_{M}=0$ which ensures that the energy density of matter remains positive as long as it is initially positive. See Fig. 7 to have an idea of how the phase maps look.

Let us analyze then each critical point for this case:

(1) $P_{0}=(0,0)$. For this critical point, the linearized system becomes

$$
\begin{aligned}
\frac{d H}{d N} \simeq & -\frac{4 \omega_{\lambda}+\sigma_{\lambda}}{2 \omega_{\lambda}+\sigma_{\lambda}+1} H \\
\frac{d x}{d N} \simeq & -\frac{2 \omega_{\lambda}+2 \sigma_{\lambda}+3}{2 \omega_{\lambda}+\sigma_{\lambda}+1} \\
& \times\left[x+\frac{2 \omega_{\lambda}-\sigma_{\lambda}-3}{2\left(2 \omega_{\lambda}+\sigma_{\lambda}+1\right)} \Omega_{M}\right] \\
\frac{d \Omega_{M}}{d N} \simeq & \frac{2 \omega_{\lambda}-\sigma_{\lambda}-3}{2 \omega_{\lambda}+\sigma_{\lambda}+1} \Omega_{M} .
\end{aligned}
$$

The eigenvalues for this system are the same as those of the vector dominance case plus $\mu_{\Omega_{M}}=$ $\left(2 \omega_{\lambda}-\sigma_{\lambda}-3\right) /\left(2 \omega_{\lambda}+\sigma_{\lambda}+1\right)$, which determines the stability of the solutions with $\Omega_{M} \rightarrow 0$. Therefore, the analysis proceeds exactly the same as before with the supplementary condition $\mu_{\Omega_{M}}<0$

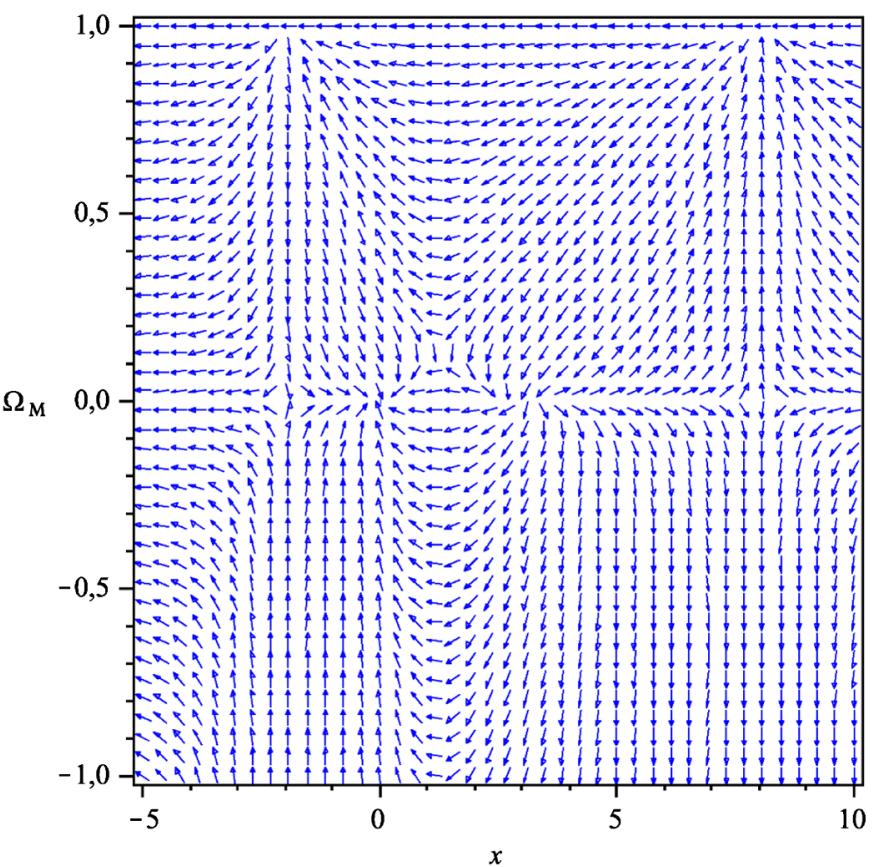

ensuring the late-time domination of the vector field. Notice that, as commented above, this condition is not the same as that given in (72). Indeed, this supplementary condition happens not to reduce the region of the parameter space in which we get attracting solutions with accelerated expansion, i.e., all the models indicated in Fig. 6 corresponding to these type of solutions have indeed $\Omega_{M}=0$ as an attractor.

(2) $P_{ \pm}=\left(x_{ \pm}, 0\right)$. In this case the linearized system becomes diagonal with the same eigenvalues for $x$ and $H$ as in the vector dominance case, i.e., $\mu_{H}=$ $-\left(2 x_{c}^{ \pm}+3\right)$ and $\mu_{x}=-2 x_{c}^{ \pm}$. Moreover, the eigenvalue for $\Omega_{M}$ is given by $\mu_{\Omega_{M}}=4 x_{c}^{ \pm}+3$. Hence, as in the case of $P_{0}$ the stability analysis is the same as that already performed above, although we must impose the condition $4 x_{c}^{ \pm}+3<0$ so that the vector field eventually dominates. However, when we impose the latter condition we find that these critical points happen not to be attractors for any value of the parameters.

So far, we have seen that the presence of a matter fluid only affects the solutions in the sense that the parameter space is restricted to that region in which $\Omega_{M}=0$ is an attractor, otherwise the vector field would never dominate and we cannot produce late-time acceleration. In other words, the features of the critical points remain the same as those studied in the vector domination case, although only the cases in the allowed region are admissible. The novelties appear when studying the separatrix.

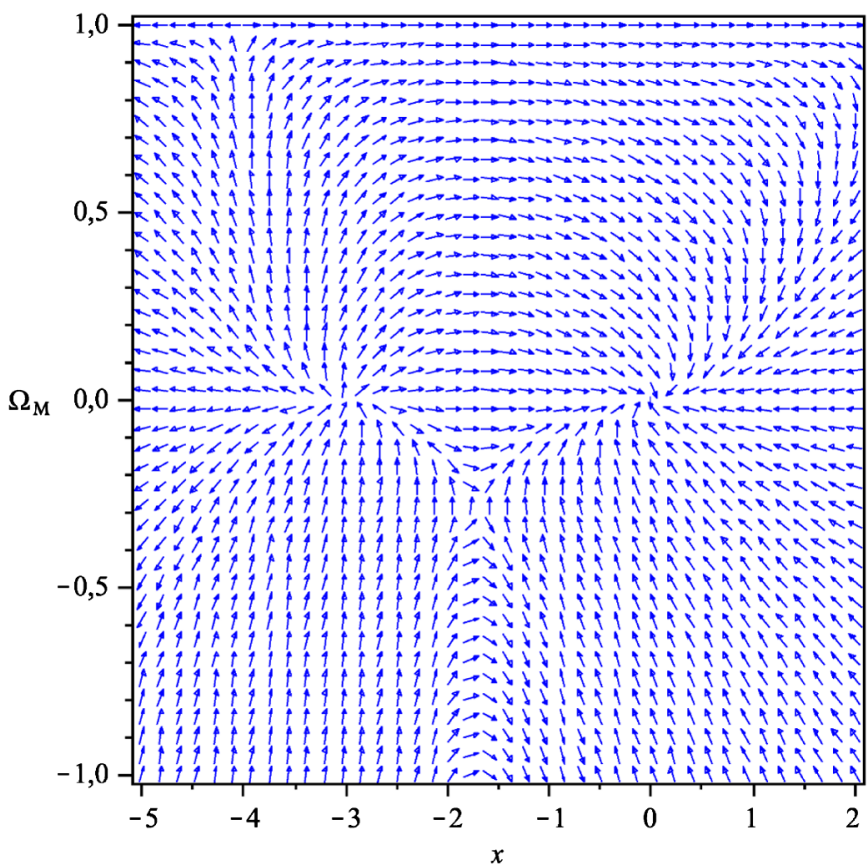

FIG. 7 (color online). In these two plots we show two examples of phase maps corresponding to the cases when the two critical points are present (left) and when they are not (right) or, equivalently, when the separatrix is open from above or from below. 
(1) Separatrix. In this case the separatrix is no longer given by $x=x_{s}$, but by the parabola

$$
\Omega_{M}=\frac{\left[x+3\left(2 \omega_{\lambda}+\sigma_{\lambda}+1\right)\right]^{2}}{\left[9\left(2 \omega_{\lambda}+\sigma_{\lambda}\right)^{2}+6\left(5 \omega_{\lambda}+2 \sigma_{\lambda}\right)\right]} .
$$

Notice that the vertex of this parabola always lies on the $x$ axis, i.e., in the vertex we always get $\Omega_{M}=0$ so that it will be interesting to have solutions attracted by it. Whether this parabola is open from above or below in the $\left(x, \Omega_{M}\right)$ plane depends on the sign of $9\left(2 \omega_{\lambda}+\sigma_{\lambda}\right)^{2}+6\left(5 \omega_{\lambda}+2 \sigma_{\lambda}\right)$ which also determines the existence of $x_{c}^{ \pm}$so that if the critical points $x_{c}^{ \pm}$exist the parabola goes up and if they do not exist the parabola goes down. Since negative values of $\Omega_{M}$ are physically unreasonable, an openfrom-below parabola does not represent a proper separatrix for the physically admissible region of the phase map.

Close to the vertex of the separatrix, i.e., for

$$
\Omega_{M}=\frac{\left[x+3\left(2 \omega_{\lambda}+\sigma_{\lambda}+1\right)\right]^{2}}{\left[9\left(2 \omega_{\lambda}+\sigma_{\lambda}\right)^{2}+6\left(5 \omega_{\lambda}+2 \sigma_{\lambda}\right)\right]}+\delta_{\Omega_{M}}
$$

$$
x=-3\left(2 \omega_{\lambda}+\sigma_{\lambda}+1\right)+\delta_{x}
$$

with $\delta_{\Omega_{M}}, \delta_{x} \ll 1$ the autonomous system becomes

$$
\begin{aligned}
\frac{d H}{d N} & \simeq 3 \frac{2 \omega_{\lambda}+\sigma_{\lambda}+1}{\delta_{\Omega_{M}}} H \\
\frac{d x}{d N} & \simeq 3\left(2 \omega_{\lambda}+\sigma_{\lambda}+1\right) \frac{\delta_{x}}{\delta_{\Omega_{M}}} \\
\frac{d \Omega_{M}}{d N} & \simeq 2 \frac{2 \omega_{\lambda}+\sigma_{\lambda}+1}{3\left(2 \omega_{\lambda}+\sigma_{\lambda}\right)^{2}+2\left(5 \omega_{\lambda}+2 \sigma_{\lambda}\right)} \frac{\delta_{x}^{2}}{\delta_{\Omega_{M}}} .
\end{aligned}
$$

In the previous expressions, $\delta_{\Omega_{M}}$ parametrizes the separation to the separatrix and is positive (negative) for points above (below) it, whereas $\delta_{x}$ gives the separation on the right $\left(\delta_{x}>0\right)$ or on the left $\left(\delta_{x}<\right.$ $0)$ from the vertex of the parabola. From the equation for $d H / d x$ we see that the trajectories will not be able to cross the vertex because $d H / d N$ becomes singular at that point. On the other hand, it is easy to see from the equations for $d x / d N$ and $d \Omega_{M} / d N$ that the vertex will always act as an attractor for the trajectories approaching from one side of the parabola so that, in the cases in which the parabola is open from above, there always exist trajectories that are attracted by the vertex of the separatrix. Whether the trajectories approaching the vertex are those going from above or below the parabola is determined by the sign of $2 \omega_{\lambda}+\sigma_{\lambda}+1$ as follows:

(a) $2 \omega_{\lambda}+\sigma_{\lambda}+1>0$. In this case the trajectories approaching from below are attracted toward the ver-

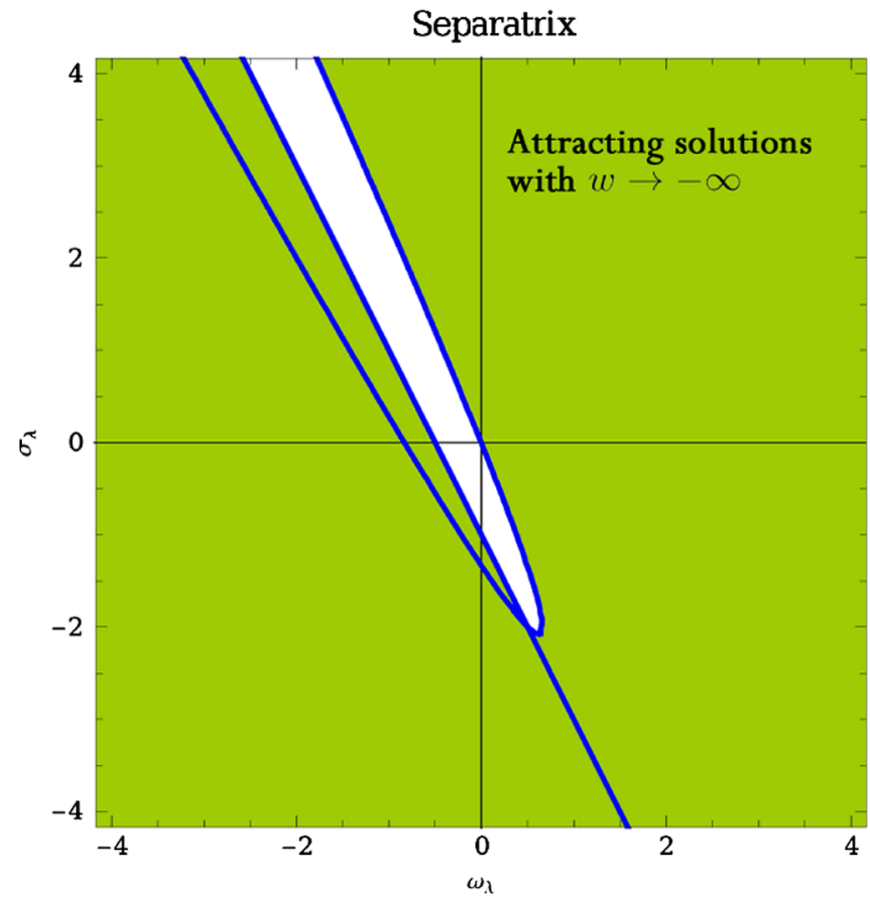

FIG. 8 (color online). In this plot we show the regions where we have the vertex of the separatrix attracting trajectories with accelerated expansion ([green] shaded region) and where there are not solutions attracted by the vertex in which the expansion is accelerated (white region). We see that, in most of the parameter space, we have that the vertex attracts some trajectories in which the equation of state of the vector field diverges evolving toward $-\infty$. The curve plotted in the graph separates those models in which the separatrix is open from above (outer region) and those in which the separatrix is open from below (inner region). Notice that these regions coincide with those in which the critical points $P_{ \pm}$exist (separatrix open from above) and they do not (separatrix open from below), as explained in the main text.

tex whereas those solutions contained in the region above the separatrix are repelled by the vertex.

(b) $2 \omega_{\lambda}+\sigma_{\lambda}+1<0$. In this case, the solutions in the region above the separatrix are attracted toward the vertex whereas the trajectories below the parabola go away from the vertex.

To study the cases when the parabola is open from below we shall analyze the autonomous systems for $x+3\left(2 \omega_{\lambda}+\right.$ $\left.\sigma_{\lambda}+1\right)=\delta_{x} \ll 1$ and $\Omega_{M} \ll 1$. In that case we obtain

$$
\begin{aligned}
\frac{d x}{d N} & \simeq 3\left(2 \omega_{\lambda}+\sigma_{\lambda}+1\right) \frac{\delta_{x}}{\Omega_{M}} \\
\frac{d \Omega_{M}}{d N} & \simeq 6\left(2 \omega_{\lambda}+\sigma_{\lambda}+1\right) .
\end{aligned}
$$

Thus, only if $\left(2 \omega_{\lambda}+\sigma_{\lambda}+1\right)<0$ the vertex of the separatrix is an attractor when the separatrix is contained in the region with $\Omega_{M}<0$.

Finally, it remains to study the behavior of the equation of state as the trajectory approaches the vertex of the separa- 
trix. If we use the parametrization given in (81) again, we find that the equation of state becomes

$$
w \simeq 2 \frac{2 \omega_{\lambda}+\sigma_{\lambda}+1}{\delta_{\Omega_{M}}} .
$$

Therefore, if the trajectory approaches the vertex from above the separatrix, the equation of state will evolve toward $+\infty(-\infty)$ as long as $2 \omega_{\lambda}+\sigma_{\lambda}+1$ is positive (negative). On the contrary, when the trajectory goes to the vertex from below the separatrix the equation of state of the vector field goes to $+\infty(-\infty)$ as long as $2 \omega_{\lambda}+\sigma_{\lambda}+1$ is negative (positive).

Then, if the parabola is open from above $\left(3\left(2 \omega_{\lambda}+\sigma_{\lambda}\right)^{2}+\right.$ $\left.2\left(5 \omega_{\lambda}+2 \sigma_{\lambda}\right)>0\right)$, irrespective of the sign of $2 \omega_{\lambda}+$ $\sigma_{\lambda}+1$ we have that the vertex acts as an attractor for some trajectories with solutions whose equation of state goes to $-\infty$. On the other hand, when the parabola is open from below $\left(3\left(2 \omega_{\lambda}+\sigma_{\lambda}\right)^{2}+2\left(5 \omega_{\lambda}+2 \sigma_{\lambda}\right)<0\right)$, only when $2 \omega_{\lambda}+\sigma_{\lambda}+1$ is negative the vertex acts as an attractor and, in that case, the equation of state in the vertex goes to $-\infty$.

Finally, we would like to comment on the existence of certain models in which we can have critical points with $\Omega_{M} \neq 0,1$. Those critical points can be found from (76) by solving $\frac{d \Omega_{M}}{d N}=0$ with respect to $x$ and for arbitrary values of $\Omega_{M}$ and, then, obtaining the corresponding critical value for $\Omega_{M}$ from the equation $\frac{d x}{d N}=0$. In these critical points, one generally gets $H=0$. The explicit expressions for these critical points are

$$
\begin{gathered}
x_{\Omega_{M}}^{ \pm}=\frac{2 \omega_{\lambda}-\sigma_{\lambda}-3 \pm \sqrt{2\left(2 \omega_{\lambda}-\sigma_{\lambda}-3\right)\left[2\left(5 \omega_{\lambda}+2 \sigma_{\lambda}\right)+3\left(2 \omega_{\lambda}+\sigma_{\lambda}\right)^{2}\right]}}{4 \omega_{\lambda}+2 \sigma_{\lambda}+1} \\
\Omega_{\bar{M}}^{ \pm}=-\frac{x_{\Omega_{M}}^{ \pm}\left[x_{\Omega_{M}}^{ \pm}+3\left(2 \omega_{\lambda}+\sigma_{\lambda}+1\right)\right]}{F_{x}\left(x_{\Omega_{M}}^{ \pm}\right)} .
\end{gathered}
$$

Therefore, only models in which $\left(2 \omega_{\lambda}-\sigma_{\lambda}-3\right) \times$ $\left[2\left(5 \omega_{\lambda}+2 \sigma_{\lambda}\right)+3\left(2 \omega_{\lambda}+\sigma_{\lambda}\right)^{2}\right]$ is positive can contain this type of critical points. Moreover, this condition does not guarantee the existence of physically admissible values for $\Omega_{M}$ because one could, in principle, obtain both positive and negative values for $\Omega_{M}$. However, these critical points cannot lead to accelerated solutions because the equation of state for the vector field in such points is identically zero, i.e., it behaves as a dust fluid.

To summarize the results of this section, we have shown that the features of the critical points for the case when we have matter in addition to the vector field remain unaffected, but the behavior of the separatrix presents novelties and, generally, in all the models we shall have attracting solutions with future singularities (see Fig. 8. Finally, we have shown that solutions in which $\Omega_{M}$ goes to some values different from 0 and 1 are such that the equation of state of the vector field goes to zero, i.e., it asymptotically behaves as a matter fluid.

\section{LOCAL GRAVITY TESTS}

The viability of any alternative theory of gravity is subject to its agreement with Solar System experiments, which provide very tight constraints on the so-called parametrized post-Newtonian (PPN) parameters. These parameters are a set of quantities that characterize most of gravity theories at small scales and are extremely useful to measure deviations from GR. For the action (2), the PPN parameters are given by [18]

$$
\begin{aligned}
\gamma & =\frac{1+4 \omega A^{2}\left(1+\frac{2 \omega+\sigma}{\epsilon}\right)}{1-4 \omega A^{2}\left(1-\frac{4 \omega}{\epsilon}\right)} \\
\beta & =\frac{1}{4}(3+\gamma)+\frac{1}{2} \Theta\left[1+\frac{\gamma(\gamma-2)}{G}\right] \\
\alpha_{1} & =4(1-\gamma)[1+2 \epsilon \Delta]+16 \omega A^{2} \Delta a \\
\alpha_{2} & =3(1-\gamma)\left[1+\frac{4}{3} \epsilon \Delta\right]+8 \omega A^{2} \Delta a-2 \frac{b A^{2}}{G} \\
\alpha_{3} & =\xi=\zeta_{1}=\zeta_{2}=\zeta_{3}=\zeta_{4}=0
\end{aligned}
$$

with

$$
\begin{gathered}
\Theta=\frac{\left(1-4 \omega A^{2}\right)(2 \epsilon+\sigma-2 \omega)}{\left(1-4 \omega A^{2}\right) 2 \epsilon+32 \omega^{2} A^{2}} \quad \Delta=\frac{1}{2 A^{2} \sigma^{2}-2 \epsilon\left[1-4 A^{2}(\omega+\sigma)\right]} \quad a=2 \epsilon(1-3 \gamma)+2 \sigma(1-2 \gamma) \\
b= \begin{cases}(2 \omega+\sigma)[(2 \gamma-1)(\gamma+1)+\Theta(\gamma-2)]-(2 \gamma-1)^{2}(2 \omega+\sigma+\lambda)\left(1-\frac{2 \omega+\sigma+\lambda}{\lambda}\right) & \lambda \neq 0 \\
0 & \lambda=0 .\end{cases}
\end{gathered}
$$


Moreover, the effective Newton constant is defined as

$$
G_{\mathrm{eff}} \equiv G\left[\frac{1}{2}(\gamma+1)+6 \omega A^{2}(\gamma-1)-2 A^{2} \sigma(1+\Theta)\right]^{-1}
$$

In the above expressions we have assumed $G_{\text {eff }}=1$ and $A$ is the value of the vector field at Solar System scales (in units of $4 \pi G)$. The parameters $(\gamma, \beta)$ are usually called the static PPN parameters and measure the space-curvature produced by a unit mass and the degree of nonlinearity relative to GR, respectively. The parameter $\xi$ measures effects of preferred location whereas $\alpha_{i}$ have to do with preferred frame effects. Finally, $\alpha_{3}$ and $\zeta_{i}$ are nonvanishing for theories in which the conservation of total momentum is violated. In GR, the PPN parameters are such that $\gamma-$ $1=\beta-1=\alpha_{1}=\alpha_{1}=\alpha_{3}=\xi=\zeta_{1}=\zeta_{2}=\zeta_{3}=\zeta_{4}=0$. On the other hand, for a general vector-tensor theory we see that there are neither preferred location effects nor violation of the total momentum conservations. However, these theories typically lead to preferred frame effects (as expected because of the presence of a vector field) as well as deviations from GR for the static PPN parameters. Current observational limits on the PPN parameters impose very stringent limits on modified gravity theories because they do not allow much deviation from GR, i.e., GR agrees with local gravity tests with very good precision [18]:

$$
\begin{array}{cc}
\gamma-1 \lesssim 2.3 \times 10^{-5} & \beta-1 \lesssim 2.3 \times 10^{-4} \\
\alpha_{1} \lesssim 10^{-4} & \alpha_{2} \lesssim 10^{-4}\left(10^{-7}\right) .
\end{array}
$$

In order to obtain constraints on the vector field from these limits we linearize the PPN parameters given in (86) as follows:

$$
\begin{aligned}
\gamma-1 & \simeq \frac{4 \omega}{\epsilon}[2(\epsilon-\omega)+\sigma] A^{2} \\
\beta-1 & \simeq \frac{(2 \omega-\sigma)(2 \epsilon-2 \omega+\sigma)\left(4 \epsilon-2 \omega+\sigma_{\lambda}\right)}{4 \epsilon^{2}} A^{2} \\
\alpha_{1} & \simeq \frac{16 \omega(2 \epsilon+\sigma)}{\epsilon} A^{2} \\
\alpha_{2} & \simeq \begin{cases}{\left[(2 \omega+\sigma)^{2}\left(\frac{1}{\epsilon}-\frac{2}{\lambda}\right)-4 \sigma\right] A^{2}} & \lambda \neq 0 \\
\frac{4 \omega}{\epsilon}[2(\epsilon+\omega)+\sigma] A^{2} & \lambda=0 .\end{cases}
\end{aligned}
$$

Therefore, we can set that, typically, the vector field at the Solar System scale will be constrained to be $A \lesssim 10^{-2}$, for models in which all the parameters are order unity. Let us remark that this value of the vector field does not need to coincide with its cosmological value.

On the other hand, the linearized Newton constant is given by

$$
G \simeq 1-\left[\frac{(2 \omega-\sigma)^{2}}{\epsilon}+4(\sigma-\omega)\right] A^{2} .
$$

Then, if we use the existent limits on its time variation $\dot{G} / G \lesssim 10^{-13} \mathrm{yr}^{-1}$ together with the constraints on the vector field obtained above, we can also set bounds on the cosmological time variation of the vector.

Although a general vector-tensor theory will be constrained by the aforementioned limits, there is a number of models whose parameters satisfy certain relations so that some of the PPN parameters could be identical to those of GR and, thus, pass the limits given in (88). Indeed, in [7] is shown that there exist a total of 6 models which are indistinguishable from GR by means of local gravity tests for any value of the background vector field, namely: $\{\sigma=$ $0, \epsilon, \lambda\}, \quad\{\sigma=-4 \lambda=-4 \epsilon\}, \quad\{\sigma=-3 \lambda=-2 \epsilon\} \quad$ and $\{\sigma=m \epsilon$, with $m=0,-2,-4\}$. Moreover, in that work, a detailed analysis of the stability of these models is performed.

\section{CONCLUSIONS AND DISCUSSION}

In this work we have developed a general study of the cosmological evolution of a vector field nonminimally coupled to gravity and without potential terms. We have given the evolution of this vector field in terms of the parameters of the theory for the different epochs of the expansion history of the Universe, namely: inflation, radiation dominated era, and matter dominated era. We have shown that it is possible to obtain a wide variety of behaviors for the evolution of the vector field by suitable choices of the parameters. In particular, we have obtained conditions for the parameters so that the vector field energy density grows or decays with respect to that of the dominant component. Moreover, conditions to have scaling evolution have also been calculated.

The case of a universe dominated by the temporal component of the vector field has been studied in detail. We have obtained an autonomous system describing the evolution of the Hubble expansion rate and the vector field. The general features of the phase map have been given and all the critical points have been appropriately characterized. For those points that act as attractors we have obtained the general conditions under which the vector field gives rise to accelerated expansion.

To study the viability of these theories as dark energy candidates we have performed an analysis of the field equations together with Einstein's equations when the Universe contains matter in addition to the vector field. Then, we have identified solutions which allow a transition from matter domination to vector domination with accelerated expansion. We have also shown that these models generally contain future singularities in which the equation of state diverges and that most of the models can give rise to periods of accelerated expansion. 
In addition to the general results commented above, we have also found a wide variety of particular model examples with interesting properties, thus: models with late-time attractors with $\Omega_{M} \neq 0,1$ all with equations of state for the vector field resembling that of nonrelativistic matter. This type of models could provide vector dark matter candidates. We have also found models with early-time accelerated solutions which are unstable thus giving rise to possible finite inflationary periods. On the other hand, the dark energy model proposed in [12] corresponds to the parameters $\epsilon=-1 / 4, \lambda=-1 / 2, \sigma=1$, $\omega=0$. Concerning the temporal component, we have that $\sigma_{\lambda}=-2$ and $\omega_{\lambda}=0$ and, if we look at Fig. 1, we see that it lies in the line of scaling behavior during radiation (what allows one to solve the coincidence problem in a natural manner) and in the growing region for the matter era, in agreement with the result found in [12]. Moreover, it belongs to the region I of the classification summarized in Table I for the vector dominance case so that $P_{0}$ and $P_{+}$ behave as attractors whereas $P_{-}$and the separatrix repel the trajectories. The singularity found in [12] corresponds to the trajectories approaching the separatrix in the case when matter is present. This is also in agreement with the results of this paper because we have that the separatrix for this particular model is given by $\Omega_{M}=\frac{1}{12}(x-3)^{2}$ so that it is open from above. Moreover, we have that $2 \omega_{\lambda}+$ $\sigma_{\lambda}+1=-1<0$ so that, according to the discussion about the separatrix in Sec. V, the trajectories above the separatrix will be attracted by its vertex. Then, as the vector field evolves as $\propto t^{\alpha}$ with $\alpha=\frac{1}{6}(-3+\sqrt{3})$ in the matter dominated era, we have that the initial conditions are given by $\Omega_{M} \simeq 1$ and $x \simeq \frac{3}{2} \alpha \simeq 0.69$ so that the cosmological solution corresponds to one of the trajectories approaching the vertex of the separatrix and, therefore, leading to a future singularity. Finally, the electromagnetic dark energy model proposed in [13] has parameters $\omega=\sigma=0, \lambda=$ $1 / 6, \epsilon=-1 / 4$ and coincides with the only model whose temporal component has constant energy density for all the cosmological epochs (see Fig. 1). Moreover, if we look at Fig. 3 we see that it belongs to case IIIa in which both critical points $P_{+}$and $P_{-}$coalesce and they coincide with the separatrix. In [13] it is shown that this model, indeed, behaves as a cosmological constant irrespective of the Hubble expansion rate so that it leads to a de Sitter universe once it dominates over the matter component.

In the last section of the paper we have given the expressions for the PPN parameters of the general vectortensor theory and used current limits on the PPN parameters to constrain the value of the vector field at small (Solar System) scales, which has been found to be $A_{\odot} \lesssim 10^{-2}$. However, one cannot, in principle, use this bound to put limits on the cosmological value of the vector field because both values do not need to coincide, unless the power spectrum of the vector field happens to be scale-invariant.
In the general case, one would need to know the mechanism that originated the primordial power spectrum of the vector field so that we can know the expectation value of the vector field at Solar System scales and, thus, compare to the experimental limits.

We would like to remark that, throughout this work, we have focused on the viability of vector-tensor theories as candidates for dark energy just for the first stage, i.e., we have given the general conditions under which the homogeneous part of a vector-tensor theory can lead to late-time acceleration. However, to propose a serious candidate one should also study the perturbations of the corresponding model and check the presence of instabilities both at classical and quantum levels. The absence of classical instabilities is required in order not to have exponentially growing modes that could spoil the predictions of the model for the zero mode. The issue of quantum instabilities has to do with the existence of modes with negative energy (ghosts) so that nonlinear interactions of the field might produce an unlimited number of such particles. The existence of such instabilities has already been studied in [7] for those vector-tensor theories which are indistinguishable from GR at small scales by considering perturbations in both the vector field and the metric. In [23], the existence of instabilities for the longitudinal component of the vector field was shown in some particular cases. In [8], a detailed treatment on the stability of the vector field perturbations for general vector-tensor theories with a potential term was performed. However, a complete study by considering metric perturbations in addition to vector perturbations remains to be done, although such a study is far from straightforward because of the large number of cases involved. In any case, as suggested in [24] where general $N$ forms are studied, the existence of singular classical solutions is related to the presence of ghosts so that one would expect that most of the vector-tensor models present instabilities (at least at the quantum level) because, as shown in this work, the existence of singular solutions at the classical level is a common feature in these models.

As a final comment, although throughout this work we have focused on vector fields as candidates for dark energy, we would like to mention that, because of their generality, the results given in the paper can also be used in other cosmological contexts in which vector fields could play a relevant role.

\section{ACKNOWLEDGMENTS}

This work has been supported by Ministerio de Ciencia e Innovación (Spain) Project Nos. FIS 2008-01323 and FPA 2008-00592, UCM-Santander PR34/07-15875, and UCMBSCH GR58/08 910309 and MEC Grant No. BES-200612059. 
[1] A. G. Riess et al., Astron. J. 116, 1009 (1998); S. Perlmutter et al., Astrophys. J. 517, 565 (1999).

[2] A. G. Riess et al., Astrophys. J. 607, 665 (2004); M. Kowalski et al., Astrophys. J. 686, 749 (2008).

[3] D. N. Spergel et al., Astrophys. J. Suppl. Ser. 148, 175 (2003); D. N. Spergel et al., Astrophys. J. Suppl. Ser. 170, 377 (2007); M. Tegmark et al., Phys. Rev. D 69, 103501 (2004).

[4] C. Wetterich, Nucl. Phys. B302, 668 (1988); R. R. Caldwell, R. Dave, and P. J. Steinhardt, Phys. Rev. Lett. 80, 1582 (1998); C. Armendariz-Picon, T. Damour, and V. Mukhanov, Phys. Lett. B 458, 209 (1999).

[5] G. Dvali, G. Gabadadze, and M. Porrati, Phys. Lett. B 485, 208 (2000); S. M. Carroll, V. Duvvuri, M. Trodden, and M. S. Turner, Phys. Rev. D 70, 043528 (2004).

[6] C. M. Will and K. Nordtvedt, Jr., Astrophys. J. 177, 757 (1972); K. Nordtvedt, Jr. and C. M. Will, Astrophys. J. 177, 775 (1972); R. W. Hellings and K. Nordtvedt, Jr., Phys. Rev. D 7, 3593 (1973).

[7] J. Beltrán Jiménez and A. L. Maroto, J. Cosmol. Astropart. Phys. 02 (2009) 025.

[8] C. Armendariz-Picon and A. Diez-Tejedor, arXiv:0904.0809.

[9] V. A. Kostelecky and S. Samuel, Phys. Rev. D 40, 1886 (1989); C. Eling, T. Jacobson, and D. Mattingly, arXiv:grqc/0410001; R. Bluhm and V. A. Kostelecky, Phys. Rev. D 71, 065008 (2005); Q. G. Bailey and V. A. Kostelecky, Phys. Rev. D 74, 045001 (2006); R. Bluhm, S. H. Fung, and V. A. Kostelecky, Phys. Rev. D 77, 065020 (2008); R. Bluhm, N. L. Gagne, R. Potting, and A. Vrublevskis, Phys. Rev. D 77, 125007 (2008).

[10] C. Eling, Phys. Rev. D 73, 084026 (2006); S. M. Carroll, T. R. Dulaney, M. I. Gresham, and H. Tam Phys. Rev. D 79, 065011 (2009); 79, 065012 (2009).

[11] C. Armendariz-Picon, J. Cosmol. Astropart. Phys. 07 (2004) 007; C. G. Boehmer and T. Harko, Eur. Phys. J. C 50, 423 (2007); M. Novello et al., Phys. Rev. D 69 , 127301 (2004); V. V. Kiselev, Classical Quantum Gravity 21, 3323 (2004); H. S. Zhao, Astrophys. J. 671, L1 (2007); K. Bamba, S. Nojiri, and S. D. Odintsov,Phys. Rev. D 77, 123532 (2008).
[12] J. Beltrán Jiménez and A.L. Maroto, Phys. Rev. D 78, 063005 (2008); arXiv:0807.2528; J. Beltrán Jiménez, Ruth Lazkoz, and A. L. Maroto, arXiv:0904.0433.

[13] J. Beltrán Jiménez and A. L. Maroto, J. Cosmol. Astropart. Phys. 03 (2009) 016; AIP Conf. Proc. 1122, 107 (2009); J. Beltrán Jiménez and A. L. Maroto, arXiv:0903.4672.

[14] L. H. Ford, Phys. Rev. D 40, 967 (1989); A. Golovnev, V. Mukhanov, and V. Vanchurin, J. Cosmol. Astropart. Phys. 06 (2008) 009; T. Koivisto and D. F. Mota, J. Cosmol. Astropart. Phys. 08 (2008) 021; S. Kanno and J. Soda, Phys. Rev. D 74, 063505 (2006); M. A. Watanabe, S. Kanno, and J. Soda, Phys. Rev. Lett. 102, 191302 (2009).

[15] M. Novello and S.E. Perez Bergliaffa, Phys. Rep. 463, 127 (2008).

[16] J. D. Bekenstein, Phys. Rev. D 70, 083509 (2004); T. G. Zlosnik, P. G. Ferreira, and G. D. Starkman, Phys. Rev. D 75, 044017 (2007).

[17] T. Koivisto and D. F. Mota, Astrophys. J. 679, 1 (2008); S. Kanno, M. Kimura, J. Soda, and S. Yokoyama, J. Cosmol. Astropart. Phys. 082008034.

[18] C. Will, Theory and Experiment in Gravitational Physics (Cambridge University Press, Cambridge, England, 1993); C. M. Will, Living Rev. Relativity 9, 3 (2006), http:// www.livingreviews.org/lrr-2006-3.

[19] T. Koivisto and D. F. Mota, J. Cosmol. Astropart. Phys. 06 (2008) 018

[20] A. L. Maroto, J. Cosmol. Astropart. Phys. 05 (2006) 015; A. L. Maroto, Int. J. Mod. Phys. D 15, 2165 (2006); AIP Conf. Proc. 878, 240 (2006); J. Beltran Jimenez and A. L. Maroto, Phys. Rev. D 76, 023003 (2007).

[21] A. Kashlinsky, F. Atrio-Barandela, D. Kocevski, and H. Ebeling, Astrophys. J. 691, 1479 (2009); arXiv:0809.3734; R. Watkins, H.A. Feldman, and M.J. Hudson, arXiv:08094041.

[22] J. D. Barrow, Phys. Rev. D 55, 7451 (1997); J. D. Barrow and R. Marteens, Phys. Rev. D 59, 043502 (1998).

[23] B. Himmetoglu, C. R. Contaldi, and M. Peloso, Phys. Rev. Lett. 102, 111301 (2009); Phys. Rev. D 79, 063517 (2009).

[24] T. S. Koivisto, D. F. Mota, and C. Pitrou, arXiv:0903.4158 [Astrophys. J. (to be published). 